\title{
FINITE TYPE 3-MANIFOLD INVARIANTS, THE MAPPING CLASS GROUP AND BLINKS
}

\author{
STAVROS GAROUFALIDIS \& JEROME LEVINE
}

\begin{abstract}
The goal of the present paper is to find higher genus surgery formulas for the set of finite type invariants of integral homology 3 -spheres, and to develop a theory of finite type invariants which will be applied in a subsequent publication [7] in the study of subgroups of the mapping class group. The main result is to show that six filtrations on the vector space generated by oriented integral homology 3-spheres (three coming from surgery on special classes of links and three coming from subgroups of the mapping class group) are equal. En route we introduce the notion of blink (a special case of a link) and of a new subgroup of the mapping class group.
\end{abstract}

\section{Introduction}

\subsection{Motivation}

The motivation/goal for the present paper is threefold:

- To find higher genus surgery formulas for the set of finite type invariants of integral homology 3 -spheres.

- To develop a theory of finite type invariants that has applications in the study of subgroups of the mapping class group.

- To propose a philosophical explanation of duality in the recent idea of $p$-branes in string theory.

Received August 22, 1996. The authors were partially supported by NSF grants DMS-95-05105 and DMS-93-03489 respectively. 
En route to achieving the above goals we came across the notion of a blink (a special kind of link, see definition 1.8) and across a new subgroup of the mapping class group; see Section 1.3.

Finite type invariants of integral homology 3-spheres, though introduced less than a year ago by $T$. Ohtsuki [22], play a crucial role in understanding the quantum invariants of 3-manifolds and may play an important role in the interaction between arithmetic, combinatorics, low dimensional topology and mathematical physics. They may also play an important role in understanding the properties of the Chern-Simons path integrals, in a way that diverges from the measure-theoretic analytic interpretation of the path integrals. And finally, they may shed some light on the obscure and not at all understood arithmetic properties of the quantum invariants of links and 3-manifolds. Finite type invariants are defined in terms of decreasing filtrations of the vector space generated by oriented integral homology 3-spheres, for a review see Section 1.2. In the present paper, there are two sources for such filtrations: one from cutting and pasting along embedded surfaces in integral homology 3-spheres, (see Section 1.3) and the other from doing surgery on (framed) links in integral homology 3-spheres (see Section 1.4). An equality of such filtrations as shown in Corollary 1.21 answers the first of the goals of the paper, and in fact characterizes finite type invariants of integral homology 3 -spheres in terms of their higher genus surgery properties.

The above equality of filtrations can be used [7] to study subgroups of the mapping class group generalizing the work of S. Morita [20], [21]. Due to the length of the present paper, we need to postpone the above study to a subsequent publication; see [7].

Finally, a word about the third motivation for the present paper: $p$ branes were introduced very recently in the physics literature; see [26]. Their role in explaining duality phenomena in string theory and field theory has been exhibited in a number of ways. Since finite type invariants are related to Chern-Simons field theory (a gauge theory in three dimensions with the Chern-Simons function as Lagrangian and (colored) knots in 3-manifolds as the observables) we may learn something about duality of gauge theories in three dimensions by studying equivalences of finite type invariants coming from surgery on one-dimensional (links) or two-dimensional (surfaces) objects in 3-manifolds. This is a valid thought since finite type invariants can be thought of as the partition function of observables of quantum field theories. 


\subsection{Finite type invariants and filtrations on $\mathcal{M}$}

All 3-manifolds are oriented and smooth and all diffeomorphisms are orientation preserving unless otherwise mentioned. Let $\mathcal{M}$ denote the vector space over $\mathbb{Q}$ on the set of orientation preserving diffeomorphism classes of integral homology 3 -spheres. Any decreasing filtration $\mathcal{F}_{*}$ on $\mathcal{M}$ defines a notion of finite type invariants of integral homology 3-spheres as follows: a map $v: \mathcal{M} \rightarrow \mathbb{Q}$ is called of $\mathcal{F}$-type $m$, if $v\left(\mathcal{F}_{m+1} \mathcal{M}\right)=0$. Examples of such filtrations were originally introduced by T. Ohtsuki [22] and by S. Garoufalidis [3]. For a review of them see Section 1.4 below.

In the present paper we describe two main sources for such filtrations: completions of the (group rings) of subgroups of the mapping class group and surgery on special classes of (framed) links. The first source is essentially two-dimensional, examples of which will be $\mathcal{F}_{*}^{T} \mathcal{M}$ and $\mathcal{F}_{*}^{K} \mathcal{M}$ and $\mathcal{F}_{*}^{\mathcal{L}} \mathcal{M}$ (described in the next Section 1.3). The second source is onedimensional, examples of which will be $\mathcal{F}_{*}^{a s} \mathcal{M}, \mathcal{F}_{*}^{b} \mathcal{M}, \mathcal{F}_{*}^{b l} \mathcal{M}$, described in Section 1.4. The equality of all such filtrations may exhibit a duality between 1-branes and 2-branes in 3-dimensional gauge field theory which has not yet been discovered by the physicists.

\subsection{Filtrations on $\mathcal{M}$ from embedded surfaces}

We begin by describing the filtrations on $\mathcal{M}$ that come from subgroups of the mapping class group. We recall first some well known facts about mapping class groups from the work of D. Johnson [12], [13] and S. Morita [20]. Let $\Gamma_{g, 1}$ denote the group of isotopy classes of orientation preserving diffeomorphisms of closed oriented genus $g$ surfaces $\Sigma_{g}$ which are the identity on a disk $D_{g} \subseteq \Sigma_{g}$. This group (which we refer to as the mapping class group) acts on the fundamental group $\pi$ of the open surface $\Sigma_{g}-D_{g}$. Note that $\pi$ is a free group in $2 g$ generators. For the sake of simplicity in notation, we will suppress the dependence of $\pi$ on $g$; we hope that this will not cause any confusion. The above discussion defines a map:

$$
\Gamma_{g, 1} \rightarrow \operatorname{Aut}(\pi)
$$

For a group $G$, let $G_{k}$ denote the lower central series, defined inductively by $G_{1}=G$, and $G_{m+1}=\left[G, G_{m}\right]$. Here for $H, K$ subsets of $G$, $[H, K]$ denotes the subgroup of $G$ generated by $[\eta, \kappa]=\eta^{-1} \kappa^{-1} \eta \kappa$, for $\eta \in H, \kappa \in K$. The above action of the mapping class group induces 
(for every non-negative integer $k$ ) an action on $\pi / \pi_{k+1}$ :

$$
\Gamma_{g, 1} \rightarrow \operatorname{Aut}\left(\pi / \pi_{k+1}\right)
$$

Let $\left(\Gamma_{g, 1}\right)_{[k]}$ denote the kernel of the above map. It is obvious that $\left\{\left(\Gamma_{g, 1}\right)_{[k]}\right\}_{k \geq 0}$ is a decreasing sequence of normal subgroups of $\Gamma_{g, 1}$. Note that $\left(\Gamma_{g, 1}\right)_{[k]}$ is denoted by $\Gamma_{g, 1}(k+1)$ in [20]. The reason for shifting the index by one in our present notation is to make the statements of question 1 (in Section 1.7) easier. Much attention has been paid to the first three members of the above sequence. The first, $\left(\Gamma_{g, 1}\right)_{[0]}$ coincides with the mapping class group $\Gamma_{g, 1}$ itself. The second, $\left(\Gamma_{g, 1}\right)_{[1]}$ is the Torelli group (i.e., the kernel of the map: $\Gamma_{g, 1} \rightarrow S p(2 g, \mathbb{Z})$, in other words all diffeomorphisms of the surface that act trivially on the homology), and will from now on be denoted by $\mathcal{T}_{g, 1}$. The third, $\left(\Gamma_{g, 1}\right)_{[2]}$ was studied extensively in [12], [13] and [20] and, following their notation, we will denote it by $\mathcal{K}_{g, 1}$.

In an alternative view, it was shown by Johnson ([12] and [13]) that $\mathcal{T}_{g, 1}$ (resp. $\mathcal{K}_{g, 1}$ ) is the subgroup of the mapping class group generated by Dehn twists on cobounding (resp. bounding) simple closed curves. We will find this alternative view very useful in the present paper. Note that all the above groups and maps behave well with respect to an inclusion of a lower genus open surface into a higher genus one, and with respect to the action of the mapping class groups given by conjugation.

Consider the lower central series subgroups $\left(\mathcal{T}_{g, 1}\right)_{n}$ or $\left(\mathcal{K}_{g, 1}\right)_{n}$ and their "rational closures" which we denote by $\left(\mathcal{T}_{g, 1}\right)_{(n)},\left(\mathcal{K}_{g, 1}\right)_{(n)}$. Here, for a group $G$ define $G_{(n)}$ to be the normal subgroup consisting of all elements $g$ such that $g^{k} \in G_{n}$ for some $k>0$. Recall that, in a nilpotent group, the set of all elements of finite order forms a normal subgroup. Thus, for every non-negative integer $n$, we can consider three interesting sequences of normal subgroups of the mapping class group: $\left(\Gamma_{g, 1}\right)_{[n]}$, $\left(\mathcal{T}_{g, 1}\right)_{n}$ and $\left(\mathcal{T}_{g, 1}\right)_{(n)}$. It was pointed out by Johnson [13] that $\left(\mathcal{T}_{g, 1}\right)_{n} \subseteq$ $\left(\Gamma_{g, 1}\right)_{[n]}$. In fact, the following, somewhat stronger, inclusion is true:

$$
\left(\mathcal{T}_{g, 1}\right)_{(n)} \subseteq\left(\Gamma_{g, 1}\right)_{[n]},
$$

because $\left(\Gamma_{g, 1}\right)_{[n]}$ is the kernel of a homomorphism from $\left(\Gamma_{g, 1}\right)_{[n-1]}$ into a torsion-free abelian group (see [13]). Note that Johnson [13] has shown that: $\left(\mathcal{T}_{g, 1}\right)_{(2)}=\mathcal{K}_{g, 1}=\left(\Gamma_{g, 1}\right)_{[2]}$. He asked whether $\left(\Gamma_{g, 1}\right)_{[n]}=\left(\mathcal{T}_{g, 1}\right)_{(n)}$ for every $n$, but this was answered in the negative by Morita [20] for $n=3$ and by Hain [11] for $n \geq 3$. 
We now introduce one more subgroup of $\Gamma_{g, 1}$ which has apparently not been considered in the literature up to now. Recall first that $H_{1}\left(\Sigma_{g}\right)$ is a symplectic $2 g$-dimensional vector space, the symplectic form being the intersection form on the homology. Let $L \subseteq H_{1}\left(\Sigma_{g}\right)$ be any $L a$ grangian, i.e., a direct summand of rank $g$ on which the intersection pairing vanishes. For example, if $M$ is an integral homology 3 -sphere, $i: \Sigma_{g} \subseteq M$ is any embedding in $M$ and $M_{ \pm}$is the closure of one of the two components of $M-i\left(\Sigma_{g}\right)$, then $\operatorname{ker}\left\{H_{1}\left(\Sigma_{g}\right) \rightarrow H_{1}\left(M_{ \pm}\right)\right\}$ is a Lagrangian. Furthermore any two Lagrangians are conjugate via an isometry of $H_{1}\left(\Sigma_{g}\right)$ and so any Lagrangian arises from an embedding this way. If $i: \Sigma_{g} \subseteq M$ is an embedding as above, then we set $L_{ \pm}^{i}=\operatorname{ker}\left\{H_{1}\left(\Sigma_{g}\right) \rightarrow H_{1}\left(M_{ \pm}\right)\right\}$, where $M_{+}$is the closure of the positive component of $M-i\left(\Sigma_{g}\right)$, i.e., the component into which the positive normal vector to $i\left(\Sigma_{g}\right)$ points, and the other component is $M_{-}$. For a fixed Lagrangian $L$ define $\mathcal{L}_{g, 1}^{L} \subseteq \Gamma_{g, 1}$ to be the subgroup generated by Dehn twists on simple closed curves representing elements of $L$; we will call these $L$-twists. Note that if $h \in \Gamma_{g, 1}$ then $\mathcal{L}_{g, 1}^{h *(L)}=h^{-1} \mathcal{L}_{g, 1}^{L} h$. Thus $\mathcal{L}_{g, 1}^{L}$ depends upon the choice of $L$, but any two choices give conjugate subgroups. Moreover $\mathcal{K}_{g, 1} \subseteq \mathcal{L}_{g, 1}^{L}$ for any choice of $L$, and the intersection of all the conjugates of $\mathcal{L}_{g, 1}^{L}$ is contained in $\mathcal{T}_{g, 1}$ since every element of $H_{1}\left(\Sigma_{g}\right)$ belongs to some Lagrangian. We will often just use the notation $\mathcal{L}_{g, 1}$ for $\mathcal{L}_{g, 1}^{L}$ when no confusion will arise. For an embedding $i: \Sigma_{g} \subseteq M$ we will use the notation $\mathcal{L}_{g, 1}^{i}=\mathcal{L}_{g, 1}^{L_{+}^{i}}$.

See the Appendix for more remarks on $\mathcal{L}_{g, 1}$.

In the present paper we will concentrate on the subgroups $\mathcal{K}_{g, 1}, \mathcal{T}_{g, 1}$ and $\mathcal{L}_{g, 1}$ of the mapping class group.

For a group $G$, let $\mathbb{Q} G$ denote the rational group algebra of $G$, and let $I G$ denote the augmentation ideal in $\mathbb{Q} G$ (generated by all elements of the form $g-1$, for all $g \in G$ ). Let us now define two decreasing filtrations on $\mathcal{M}$ as follows: Let $M$ be an integral homology 3-sphere and $i: \Sigma \hookrightarrow M$ an embedded, oriented, connected, separating genus $g$ surface in $M$. Such a surface will be called admissible in $M$. Given any element $f$ of the mapping class group of $\Sigma$, let $M_{f}$ denote the 3-manifold obtained by cutting $M$ along $\Sigma$, twisting by $f$ and gluing back. If $f \in \mathcal{L}_{g, 1}^{i}$ and $M$ is an integral homology 3 -sphere, then it is easy to see that the resulting manifold will also be an integral homology 3-sphere. The assignment $f \rightarrow M_{f}$ defines maps $\mathbb{Q L}_{g, 1}^{i} \rightarrow \mathcal{M}$ and $\mathbb{Q} \mathcal{T}_{g, 1} \rightarrow \mathcal{M}$. We will be interested in their restrictions to the $m^{\text {th }}$ 
power of the augmentation ideals (for every non-negative integer $m$ ):

$$
\left(I \mathcal{L}_{g, 1}^{i}\right)^{m} \rightarrow \mathcal{M}, \quad\left(I \mathcal{T}_{g, 1}\right)^{m} \rightarrow \mathcal{M} \text { and } \quad\left(I \mathcal{K}_{g, 1}\right)^{m} \rightarrow \mathcal{M}
$$

We now propose the following three filtrations on $\mathcal{M}$ :

Definition 1.1. Let, respectively, $\mathcal{F}_{m}^{\mathcal{L}} \mathcal{M}, \mathcal{F}_{m}^{T} \mathcal{M}$ and $\mathcal{F}_{m}^{K} \mathcal{M}$ denote the span of the images of the above maps for all admissible surfaces $\Sigma$ in all integral homology 3-spheres $M$.

For the sake of motivation, we make a few remarks which will be proved later in Sections 2.1 and 2.3:

Remark 1.2. We will show, in Theorem 1, that the filtration $\mathcal{F}_{m}^{K} \mathcal{M}$ is equivalent to one considered by the first author in [3]. The other ones are apparently new.

We now show how to describe these filtrations using only Heegaard embeddings, i.e., embeddings whose complementary components $M_{+}$ and $M_{-}$are handlebodies. For each $g \geq 0$ choose a Heegaard embedding $i_{g}: \Sigma_{g} \hookrightarrow S^{3}$ and consider the associated maps: $\left(I \mathcal{T}_{g, 1}\right)^{m} \rightarrow \mathcal{M}$ and $\left(I \mathcal{K}_{g, 1}\right)^{m} \rightarrow \mathcal{M}$.

Definition 1.3. Let $\mathcal{F}_{m}^{H T} \mathcal{M}$ and $\mathcal{F}_{m}^{H K} \mathcal{M}$ denote the union of the spans, over the chosen $i_{g}$, of the images of the maps, as defined above, $\left(I \mathcal{T}_{g, 1}\right)^{m} \rightarrow \mathcal{M}$ and $\left(I \mathcal{K}_{g, 1}\right)^{m} \rightarrow \mathcal{M}$, respectively.

The filtration $\mathcal{F}_{m}^{\mathcal{L}} \mathcal{M}$ is more complicated to describe. If $i: \Sigma \subseteq M$ is a Heegaard embedding and $L \subseteq H=H_{1}\left(\Sigma_{g}\right)$ is a Lagrangian, we will say that $i, L$ are compatible if $L=\left(L \cap L_{+}^{i}\right)+\left(L \cap L_{-}^{i}\right)$, where we recall that $L_{ \pm}^{i}=\operatorname{ker}\left\{i_{ \pm *}: H \rightarrow H_{1}\left(M_{ \pm}\right)\right\}$and $i_{ \pm}: \Sigma_{g} \hookrightarrow M_{ \pm}$are the inclusions.

Suppose that $h$ is any orientation-preserving diffeomorphism of $\Sigma$. Then it is easy to see that $L_{ \pm}^{i h^{-1}}=h_{*}\left(L^{i}\right)$. Thus $i, L$ are compatible if and only if $i h^{-1}, h_{*}(L)$ are compatible.

Proposition 1.4. If $i, L$ are compatible and $h \in \mathcal{L}_{g, 1}^{L}$, then $M_{h}$ is an integral homology 3-sphere.

Definition 1.5. For each genus $g$ choose a Heegaard embedding $i_{g}: \Sigma_{g} \subseteq S^{3}$. Let $\mathcal{F}_{m}^{H \mathcal{L}} \mathcal{M}$ denote the union of the span, over all $g$ and all $L$ compatible with $i_{g}$, of the images of $\left(I \mathcal{L}_{g, 1}^{L}\right)^{m} \rightarrow \mathcal{M}$.

Proposition 1.6. The filtrations of $\mathcal{M}$ defined for Heegaard embeddings in definitions 1.3 and 1.5 are the same as those defined for all admissible embeddings in definition 1.1 
These propositions will be proved in Section 2.3.

Remark 1.7. We mention another set of related, and perhaps equal, filtrations. Consider the lower central series subgroups $\left(\mathcal{T}_{g, 1}\right)_{m}$ or $\left(\mathcal{K}_{g, 1}\right)_{m}$ and their "rational closures" $\left(\mathcal{T}_{g, 1}\right)_{(m)},\left(\mathcal{K}_{g, 1}\right)_{(m)}$. We will show in Section 2.1 that $\mathcal{F}_{m}^{T} \mathcal{M}$ (respectively $\mathcal{F}_{m}^{K} \mathcal{M}$ ) contains the subspace of $\mathcal{M}$ spanned by elements of the form $M-M_{f}$ for all admissible surfaces $f: \Sigma \rightarrow M$ where $f \in\left(\mathcal{T}_{g, 1}\right)_{(m)}$ (respectively $\left.\left(\mathcal{K}_{g, 1}\right)_{(m)}\right)$. But these subspaces also define filtrations of $\mathcal{M}$ worth considering.

\subsection{Filtrations on $\mathcal{M}$ from framed links}

In this section we recall filtrations on $\mathcal{M}$ from special classes of framed links (algebraically split and boundary) in integral homology 3-spheres, and we introduce yet another filtration from what we shall call blinks. We begin by recalling some definitions from [22] and [3].

A link $L$ in a 3 -manifold $M$ is called algebraically split if the linking numbers between its components vanish. A link is called boundary if each component bounds an oriented surface (often called Seifert), such that these Seifert surfaces are all disjoint from each other. Of course, the Seifert surfaces are not unique. Let $|L|$ denote the number of components of a link $L$. A framing of a link $L$ in an integral homology 3 -sphere $M$ is a choice of (isotopy class) of an essential simple closed curve in the boundary of a tubular neighborhood of each of its components. Since $M$ is an integral homology 3 -sphere, then a framing $f$ on a $r$ component link can be described in terms of a sequence $f=\left(f_{1}, \cdots, f_{r}\right)$, where $f_{i} \in \mathbb{Q} \cup\{1 / 0\}$, with the convention that $f_{i}=p_{i} / q_{i}$ is the isotopy class of the curve $p_{i}$ (meridian $)+q_{i}$ (longitude). Note that a framing of a link does not require the choice of an orientation of it. A unit framing $f$ of a link in an integral homology 3 -sphere is one such that $f_{i} \in\{-1,1\}$ for all $i$. A link is called $A S$-admissible (respectively, B-admissible) if it is algebraically split (respectively, boundary) and unit-framed. It is obvious that $B$-admissible links are $A S$-admissible. The converse is obviously false, as the Whitehead link shows. If $(L, f)$ is a framed link in a 3-manifold $M$, we denote by $M_{L, f}$ the 3 -manifold obtained by doing Dehn surgery to each component of the framed link $L$. Let $\mathcal{M}$ denote the set of integral homology 3 -spheres. For an $A S$-admissible link $(L, f)$ in an integral homology 3 -sphere $M$, let

$$
[M, L, f]=\sum_{L^{\prime} \subseteq L}(-1)^{\left|L^{\prime}\right|} M_{L^{\prime}, f^{\prime}} \in \mathcal{M}
$$


where the sum is over all sublinks of $L$ (including the empty one), $f^{\prime}$ is the restriction of the framing $f$ of $L$ to $L^{\prime}$, and $\left|L^{\prime}\right|$ is the number of components of $L^{\prime}$. Note that since $(L, f)$ is an $A S$-admissible link, $M_{L^{\prime}, f^{\prime}}$ is an integral homology 3-sphere for every sublink $L^{\prime}$ of $L$.

Let $\mathcal{F}_{m}^{a s} \mathcal{M}$ (respectively, $\mathcal{F}_{m}^{b} \mathcal{M}$ ) denote the subspace of $\mathcal{M}$ spanned by $[M, L, f]$ for all $A S$-admissible (respectively $B$-admissible) $m$ component links $L$ in integral homology 3 -spheres $M$. It is obvious that $\mathcal{F}_{*}^{a s} \mathcal{M}, \mathcal{F}_{*}^{b} \mathcal{M}$ are decreasing filtrations on the vector space $\mathcal{M}$. Following Ohtsuki [22] and Garoufalidis [3] we call a map $v: \mathcal{M} \rightarrow \mathbb{Q}$ an $A S$ type (respectively $B$-type) $m$ invariant of integral homology 3 -spheres if $v\left(\mathcal{F}_{m+1}^{a s} \mathcal{M}\right)=0$ (respectively, $\left.v\left(\mathcal{F}_{m+1}^{b} \mathcal{M}\right)=0\right)$.

We now present an important definition for the present paper.

Definition 1.8. A blink $L=L_{b l}$ in an integral homology 3-sphere $M$ is a link with the following properties:

- The components of $L_{b l}$ are partitioned into classes of two components each. These classes will be called the pairs of $L_{b l}$.

- $L_{b l}$ is an oriented link.

- The pairs $\{p\}$ bound disjoint oriented surfaces $\left\{\Sigma_{p}\right\}$ in $M$ (called Seifert surfaces of $\left.L_{b l}\right)$ so that, if $p=\left(l, l^{\prime}\right)$, then $\partial \Sigma_{p}=l-l^{\prime}$ (using the orientations of $l$ and $l^{\prime}$ ).

An example is given in Figure 1. An $r$-pair blink $L_{b l}$ is one such that $\left|L_{b l}\right|=2 r$. A subblink $L_{b l}^{\prime}$ of a blink $L_{b l}$ is a sublink $L_{b l}^{\prime}$ of $L_{b l}$ which is a union of some of the pairs of $L_{b l}$. Thus an $r$-pair blink $L_{b l}$ has $2^{r}$ subblinks.

We next discuss admissible framings of blinks. Recall that every component of a blink is oriented (as a knot). A zero Seifert-framing of a blink is the (isotopy class of a) parallel of it in the Seifert surface that the blink bounds. The result is independent of the Seifert surface chosen, and depends only on the orientation of the blink. A zero Seifertframing (together with the orientation of the blink) defines, for every choice of a pair of integers $(n, m)$, an $(n, m)$ Seifert-framing of a 1-pair blink. A unit Seifert-framing of a 1-pair blink is a $(\epsilon,-\epsilon)$ Seifert-framing, where $\epsilon= \pm 1$. A unit Seifert-framing of a blink is the choice of a unit Seifert-framing to each of its pairs. A blink is called $B L$-admissible if it is unit Seifert-framed.

Remark 1.9. Every $r$-component link in an integral homology 3sphere has a zero framing, which, for a choice of integers $\left(f_{1}, \cdots, f_{r}\right)$ 
defines a $\left(f_{1}, \cdots, f_{r}\right)$-framing. For a 1-pair blink, an $(n, m)$ Seifertframing is equal to a $\left(n+l_{12}, m+l_{12}\right)$ framing of it, where $l_{12}$ is the linking number between the two components of the blink. Note that a blink is not necessarily an algebraically split link. The two components of a pair may have a non-zero linking number, and components of different pairs may also have non-zero linking number.

Remark 1.10. If a unit-framed link $(L, f)$ is included (as a disjoint union of simple closed curves) in the image of an embedded surface $i: \Sigma \hookrightarrow M$, then $M_{L, f}=M_{i, \tau(L, f)}$ where $\tau(L, f)$ is an $f$-dependent product of Dehn twists along the simple closed curves on $\Sigma$ represented by $L$.

For a $B L$-admissible $r$-pair blink $L_{b l}$ in an integral homology 3sphere $M$, we denote

$$
\left[M, L_{b l}, f\right]=\sum_{L_{b l}^{\prime}}(-1)^{1 / 2\left|L_{b l}^{\prime}\right|} M_{L_{b l}^{\prime}, f^{\prime}} \in \mathcal{M}
$$

where the sum is over all subblinks $L_{b l}^{\prime}$ of $L_{b l}$ (including the empty one), and $f^{\prime}$ is the restriction of the framing $f$ of $L_{b l}$ to $L_{b l}^{\prime}$. The above definition makes sense (i.e., each 3-manifold obtained by surgery on some pairs of the blink is an integral homology 3 -sphere) because of the following lemma:

Lemma 1.11. If $\left(L_{b l}, f\right)$ is a $B L$-admissible blink in an integral homology 3-sphere $M$, then $M_{L_{b l}, f}$ is an integral homology 3-sphere.

Proof. Since the order of the first homology of $M_{L_{b l}, f}$ is the absolute values of the determinant of the linking matrix of $L_{b l}$, we only need to check that the linking matrix of $L_{b l}$ is unimodular. We proceed by induction on the number of pairs of $|L|$. If $L_{b l}=\left(L_{1}, L_{2}\right)$ is a 1-pair blink, consisting of two components $L_{1}$ and $L_{2}$ with unit Seifert-framing, then the linking matrix of $L_{b l}$ is:

$$
\left(\begin{array}{cc}
l_{12}+\epsilon & l_{12} \\
l_{12} & l_{12}-\epsilon
\end{array}\right)
$$

where $l_{12}$ is the linking number between $L_{1}$ and $L_{2}$ (which does not necessarily vanish) and $\epsilon= \pm 1$. It is clear that this is a unimodular matrix. In general, if $L_{b l}=L_{b l}^{\prime} \cup\left(L_{1}, L_{2}\right)$ is an $r$-pair blink which is the union of an $r-1$ pair blink (with unimodular linking matrix $A$ ) and a 
1-pair blink, then the linking matrix of $L_{b l}$ is:

$$
\left(\begin{array}{ccccc} 
& & & a_{1} & a_{1} \\
& A & & \vdots & \vdots \\
& & & a_{m-1} & a_{m-1} \\
a_{1} & \ldots & a_{m-1} & k_{m}+\epsilon & k_{m} \\
a_{1} & \ldots & a_{m-1} & k_{m} & k_{m}-\epsilon
\end{array}\right) .
$$

We leave it as an exercise for the reader to show that this matrix is unimodular. q.e.d.

We denote by $\mathcal{F}_{m}^{b l} \mathcal{M}$ the subspace of $\mathcal{M}$ spanned by $\left[M, L_{b l}, f\right]$ for all $B L$-admissible $m$-pair blinks $L_{b l}$ in integral homology 3 -spheres $M$. It is obvious that $\mathcal{F}_{*}^{b l} \mathcal{M}$ is a decreasing filtration on the vector space $\mathcal{M}$. We call a map $v: \mathcal{M} \rightarrow \mathbb{Q}$ a $B L$-type $m$ invariant of integral homology 3 -spheres if $v\left(\mathcal{F}_{m+1}^{b l} \mathcal{M}\right)=0$.

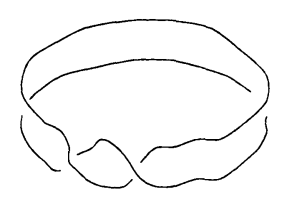

Figure 1. An unblink bounding a genus 0 surface.

Remark 1.12. The motivation and usefulness of the above notion of blink comes from several facts.

- Blinks are closely related to bounding pairs of simple closed curves in an embedded oriented surface in the 3-manifold; see Theorem 1. We will show that the filtration $\mathcal{F}_{*}^{b l} \mathcal{M}$ on $\mathcal{M}$ coming from blinks is equal to the filtration $\mathcal{F}_{*}^{T} \mathcal{M}$ coming from the $I$-adic completion of the Torelli group, in much the same way that (see Remark 1.2) the filtration $\mathcal{F}_{*}^{b} \mathcal{M}$ coming from boundary links is equal to the filtration $\mathcal{F}_{*}^{K} \mathcal{M}$; see Theorem 1 .

- Johnson [12] proved that Dehn twists on bounding pairs of simple closed curves generate the Torelli group and that Dehn twists on bounding closed curves generate $\mathcal{K}_{g, 1}$.

Remark 1.13. For later reference, let us point out that $\mathcal{F}_{m}^{b} \mathcal{M} \subseteq$ $\mathcal{F}_{m}^{b l} \mathcal{M}$. Indeed, given any framed boundary link we can convert it to a blink by punching a small hole in each Seifert surface and putting the 
appropriate unit framing on the boundary of the hole. Framed surgery on this blink obviously gives the same result as surgery on the original boundary link.

Remark 1.14. We claim that $\mathcal{F}_{m}^{b l} \mathcal{M}$ can be generated by $\left[M, L_{b l}, f\right]$ for $B L$-admissible $m$-pair blinks such that each pair bounds a genus-1 surface. Indeed, this follows from the following identity (and induction on the genus) where $L_{1}, L_{2}, K_{1}, K_{2}$ are as in Figure 2:

$$
\left[M, L_{b l}, f\right]=M-M_{L_{1}, L_{2}}=\left[M, L_{b l}^{1}, f^{1}\right]+\left[M_{L_{b l}^{1}}, L_{b l}^{2}, f^{2}\right]
$$

where $L_{b l}=\left(L_{1}, L_{2}\right), L_{b l}^{1}=\left(L_{1}, K_{1}\right)$ and $L_{b l}^{2}=\left(K_{2}, L_{2}\right)$ are unit Seifertframed 1-pair blinks.

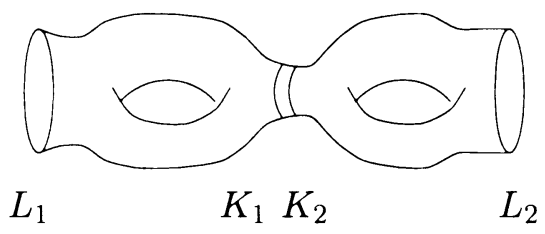

Figure 2. Shown here is a 1-pair blink $\left(L_{1}, L_{2}\right)$ of genus 2, and two (parallel) knots $K_{1}, K_{2}$ on the Seifert surface that it bounds. The knots separate the surface in two genus-1 surfaces, and are oriented and framed in an opposite way.

We will find later on the following notation useful.

Definition 1.15. If $\mathcal{N}$ is any subspace of $\mathcal{M}$, then

$$
\widehat{\mathcal{N}}=\cap_{n}\left(\mathcal{N}+\mathcal{F}_{n}^{a s} \mathcal{M}\right) .
$$

At this point we have introduced six filtrations on $\mathcal{M}$ :

$$
\mathcal{F}_{m}^{T} \mathcal{M}, \mathcal{F}_{m}^{K} \mathcal{M}, \mathcal{F}_{m}^{\mathcal{L}} \mathcal{M}, \mathcal{F}_{m}^{a s} \mathcal{M}, \mathcal{F}_{m}^{b} \mathcal{M}, \mathcal{F}_{m}^{b l} \mathcal{M}
$$

The purpose of the paper is to show, among other things, that the six associated filtrations

$$
\mathcal{F}_{m}^{T} \mathcal{M}, \widehat{\mathcal{F}_{m}^{K} \mathcal{M}}, \mathcal{F}_{m}^{\mathcal{L}} \mathcal{M}, \mathcal{F}_{m}^{a s} \mathcal{M}, \widehat{\mathcal{F}_{m}^{b} \mathcal{M}}, \mathcal{F}_{m}^{b l} \mathcal{M}
$$

are actually equal (after renumbering); see Corollary 1.21. 


\subsection{Statement of the results}

We are now ready to formulate the main results of the paper which consist of three parts. The division in three parts is for the convenience of the reader, since the methods used in each part are very different.

In the first part, we compare mapping class group filtrations and link filtrations as follows:

Theorem 1. For every non-negative integer $m$ we have:

(a) $\mathcal{F}_{m}^{T} \mathcal{M}=\mathcal{F}_{m}^{b l} \mathcal{M}$,

(b) $\mathcal{F}_{m}^{K} \mathcal{M}=\mathcal{F}_{m}^{b} \mathcal{M}$,

(c) $\mathcal{F}_{m}^{\mathcal{L}} \mathcal{M}=\mathcal{F}_{m}^{a s} \mathcal{M}$

as subspaces of $\mathcal{M}$.

A part of the argument used in the proof of Theorem 1 will also yield the following interesting fact.

Proposition 1.16. Every integral homology 3-sphere can be obtained by surgery on a boundary link in $S^{3}$.

The above proposition (which, as the referee informs us, was already known to C. Lescop using a different argument) implies the following corollary:

Corollary 1.17. The Casson invariant $\lambda_{C}$ of an integral homology 3-sphere (which we may assume is diffeomorphic to $S_{L, f}^{3}$ for a unitframed boundary $r$-component link L) is given by

$$
\lambda_{C}(M)=\sum_{i=1}^{r} f_{i} \phi\left(L_{i}\right),
$$

where $\left\{L_{i}\right\}$ are the components of the link, and $\phi\left(L_{i}\right)$ is the second derivative of the (normalized) Alexander polynomial of the knot $L_{i}$; see [14]. The point, of course, is that the Casson invariant of an integral homology 3-sphere can, therefore, be calculated in terms of the associated knot invariant (i.e., the second derivative of the normalized Alexander polynomial).

A generalization of the above corollary appears in [4].

In the second part we compare the three filtrations $\mathcal{F}_{*}^{a s} \mathcal{M}, \mathcal{F}_{*}^{b} \mathcal{M}$ and $\mathcal{F}_{*}^{b l} \mathcal{M}$ coming from special classes of links in integral homology 3 -spheres. We have the following results: 
Theorem 2. For every non-negative integer $m$ we have that:

$$
\mathcal{F}_{2 m-1}^{b l} \mathcal{M} \subseteq \mathcal{F}_{3 m}^{a s} \mathcal{M}
$$

as subspaces of $\mathcal{M}$.

Corollary 1.18. Let $v$ be an AS-type $3 m$ invariant of integral homology 3-spheres, $M$ an integral homology 3-sphere and $\Sigma$ an oriented embedded genus $g$ surface. Let $f \in\left(\mathcal{T}_{g, 1}\right)_{(2 m+1)}$ be an element of the rational closure of $\left(\mathcal{T}_{g, 1}\right)_{2 m+1}$ in the Torelli group, as in Remark 1.7(b). Then, using that remark, we have that $v(M)=v\left(M_{f}\right)$.

Theorem 3. There is an increasing function $f: \mathbb{N} \rightarrow \mathbb{N}$ such that for every non-negative integer $m$ we have that:

$$
\mathcal{F}_{f(m)}^{a s} \mathcal{M} \subseteq \mathcal{F}_{m}^{b l} \mathcal{M}
$$

Remark 1.19. In fact, since $\mathcal{F}_{*}^{a s} \mathcal{M}$ and $\mathcal{F}_{*}^{b l} \mathcal{M}$ are decreasing filtrations, the proof of Theorem 3 shows that we can take $f(m)=\mathrm{cm}^{13}$, for some positive integer constant $c$.

Remark 1.20. En route to proving Theorem 3 we give, in Proposition 3.14 , a 4 -term relation that holds in $\mathcal{M}$. The relation is apparently new. The relation between the 4-term and existing relations on $\mathcal{M}$ (namely the $A S$ and the $I H X$, see [9]) is addressed in question 2.

\section{Theorem 4.}

- With the notation of definition 1.15 , for every $m$ we have:

$$
\mathcal{F}_{3 m}^{a s} \mathcal{M} \subseteq \widehat{\mathcal{F}_{2 m}^{b l} \mathcal{M}}
$$

- Together with Theorem 2 and Theorem 3 this implies that, for every $m$ :

$$
\mathcal{F}_{2 m}^{b l} \mathcal{M}=\mathcal{F}_{2 m-1}^{b l} \mathcal{M}=\mathcal{F}_{3 m}^{a s} \mathcal{M}
$$

Combining Theorem 4 and Theorem 2 from [6], we obtain the following corollary:

Corollary 1.21. For every non-negative integer $m$, the six filtrations on $\mathcal{M}$ shown below are equal: 


\begin{tabular}{|c|c|c|}
\hline$\widehat{\mathcal{F}_{m}^{K} \mathcal{M}}$ & $\mathcal{F}_{2 m}^{T} \mathcal{M}$ & $\mathcal{F}_{3 m}^{\mathcal{L}} \mathcal{M}$ \\
\hline$\widehat{\mathcal{F}_{m}^{b} \mathcal{M}}$ & $\mathcal{F}_{2 m}^{b l} \mathcal{M}$ & $\mathcal{F}_{3 m}^{a s} \mathcal{M}$ \\
\hline
\end{tabular}

Note that in the top row the three filtrations on $\mathcal{M}$ come from subgroups of the mapping class group, and on the bottom row are their equivalent filtrations that come from special classes of links (boundary, blinks and algebraically split).

In the third part of the paper we discuss the relation between blink surgery equivalence and the Seifert matrix of a blink. In [22] and [5] it is observed that if two $A S$-admissible $n$-component links are surgery equivalent, then the associated elements in $\mathcal{G}_{n}^{a s} \mathcal{M}$ are equal. Since it is known [19] that surgery equivalence is determined by the triple $\bar{\mu}$ invariants of Milnor, these numerical link invariants provide a set of generators of $\mathcal{G}_{n}^{a s} \mathcal{M}$. We now present an analogous result for blinks and $\mathcal{G}_{n}^{b l} \mathcal{M}$. This gives, as a consequence, a set of generators for $\mathcal{G}_{n}^{b l} \mathcal{M}$. Although $\mathcal{G}_{2 n}^{b l} \mathcal{M}=\mathcal{G}_{3 n}^{a s} \mathcal{M}$, the above mentioned two sets of generators seem to be different. We hope to explore this point further some time in the near future.

Definition 1.22. Let $(M, L)$ be a blink. An elementary (blink) surgery equivalence on $(M, L)$ is a surgery on a unit Seifert-framed blink $L^{\prime \prime} \subseteq M-L$ such that $L$ and $L^{\prime \prime}$ have Seifert surfaces which are disjoint from each other. (Thus $L \cup L^{\prime \prime}$ is a blink in $M$ ). We say $\left(M_{L^{\prime \prime}}, L\right.$ ) is surgery equivalent to $(M, L)$. More generally, surgery equivalence is the equivalence relation among blinks generated by elementary surgery equivalence.

Theorem 5. Two blinks are surgery equivalent if and only if they admit equal Seifert matrices.

\subsection{Plan of the proof}

As mentioned in the abstract and the introduction, for the convenience of the reader, the proofs of the results appear in three sections.

In Section 2 we review $I$-adic and nilpotent completions and prove the claims in Remark 1.7, as well as Theorem 1 and Propositions 1.4, 1.6 and 1.16. The proofs in this section are mostly algebraic manipulations in group algebras and a bit of cutting, pasting and tubing arguments.

In Section 3.1 we review the main identities in $\mathcal{M}$ in graphical and algebraic form, as well as a few facts about blinks. There is a plethora 
of identities on $\mathcal{M}$, coming from Kirby calculus, that is, from different ways of representing an integral homology 3-sphere by surgery on a link. Most of the identities are known, however in Sections 3.2 and 3.3 we introduce a new one (the 4-term relation) that will be used crucially in the present paper.

The identities of Section 3.1 together with induction are the main tools used in the proofs of Theorems 2, 3 and 4. We warn the reader that though the statements of the above mentioned theorems seem similar, the methods used to prove them are different. Each theorem requires its own use of the same identities.

In Section 4 we discuss the notion of blink surgery equivalence and prove Theorem 5 .

We collect in the appendix some results related to the subgroup $\mathcal{L}_{g, 1}$ of the mapping class group. These results are not directly used in the present paper, but they may help clarify the structure of $\mathcal{L}_{g, 1}$.

\subsection{Questions}

In this section we propose a few questions that may lead to a better understanding of the subject. They are addressed to different audiences, at least the way they are stated.

Question 1. Are the subspaces of $\mathcal{M}$ (defined in Remark 1.2) spanned by elements of the form $M-M_{f}$, for $(M, \Sigma)$ admissible and $f \in$ $\left(\mathcal{L}_{g, 1}\right)_{(m)},\left(\mathcal{T}_{g, 1}\right)_{(m)}$ or $\left(\mathcal{K}_{g, 1}\right)_{(m)}$, respectively, the same as $\mathcal{F}_{m}^{\mathcal{L}} \mathcal{M}, \mathcal{F}_{m}^{T} \mathcal{M}$ or $\mathcal{F}_{m}^{K} \mathcal{M}$, respectively?

Question 2. In Sections 3.2 and 3.3 we show (three versions of) a 4 -term relation that holds on $\mathcal{M}$. In the first version this relation really comes from the 4 -term relation on the space of knots (in $S^{3}$ ) via the Dehn surgery map. On the other hand, one knows that an antisymmetry $(A S)$ and an $I H X$ relation hold on $\mathcal{M}$, see [9]. Is it true that the $A S$ and the $I H X$ relation are equivalent to the 4 -term relation on $\mathcal{M}$ ? Note that the $A S$ and the $I H X$ relation are equivalent to the 4-term relation on the space of knots; see [1].

Question 3. The subgroup $\mathcal{L}_{g, 1}$ of the mapping class group which is introduced in Section 1.3 is related to a larger subgroup $\overline{\mathcal{L}}_{g, 1}$ consisting of all $h \in \Gamma_{g, 1}$ such that $h \mid L=$ identity. In the appendix we discuss these two subgroups and, for example, show that $\overline{\mathcal{L}}_{g, 1} \neq \mathcal{L}_{g, 1}$. But is the filtration of $\mathcal{M}$ defined by the powers $\left(I \overline{\mathcal{L}}_{g, 1}\right)^{m}$ different from that defined by $\left(I \mathcal{L}_{g, 1}\right)^{m}$ ? 
In the appendix we also show that $\left(\overline{\mathcal{L}}_{g, 1}\right)_{5} \subseteq \mathcal{K}_{g, 1}$ but $\left(\overline{\mathcal{L}}_{g, 1}\right)_{4} \nsubseteq \mathcal{K}_{g, 1}$. Is $\left(\mathcal{L}_{g, 1}\right)_{4} \subseteq \mathcal{K}_{g, 1}$ ?

\subsection{Philosophical comments}

In this section we propose a few questions of philosophical interest that may lead to a better understanding of the subject. They are not directly related to the results of the present paper, and the questions themselves are somewhat vague. Positive answers may nevertheless bring presents to a variety of areas.

Question 4. Interpret the results of the present paper in terms of the not-yet-discovered duality of three-dimensional gauge theories.

Question 5. Give a Hodge structure on the complexified space $\mathcal{M} \otimes_{\mathbb{Q}} \mathbb{C}$ in a way compatible with the filtration discussed in the present paper.

\section{Acknowledgment}

We wish to thank the Internet for providing the required communications. The first author wishes to thank D. Bar-Natan for intriguing conversations that lead to some of the results of the present paper.

\section{Equivalence of filtrations from links and from surfaces}

\section{1. $I$-adic and nilpotent completions}

In this section we recall some facts about the lower central series of a group and its relation to the $I$-adic filtration of the group algebra. Let $G$ be a group and $\mathbb{Q} G$ the group $\mathbb{Q}$-algebra, i.e., the vector space over $\mathbb{Q}$ with $G$ as basis. Multiplication is defined by linearly extending the multiplication of $G$. The augmentation ideal $I G \subseteq \mathbb{Q} G$ is the twosided ideal generated by all elements of the form $g-1, g \in G$. The $I$-adic filtration of $\mathbb{Q} G$ is the sequence of powers $(I G)^{n}$ of $I G$. It is not difficult to show that if $g \in G_{n}$ the $n$-th lower central series subgroup of $G$ (see Section 1.3), then $g-1 \in(I G)^{n}$. In fact a bit more is true, namely let $G_{(n)}$ denote the rational closure of the group $G_{n}$ defined in Remark 1.7. Then $g-1 \in(I G)^{n}$ whenever $g \in G_{(n)}$. This follows from the fact that $g^{m}-1 \in(I G)^{n}$ for some $m$, and from the formula: 
$g^{m}-1=\sum_{i=1}^{m}\left(\begin{array}{c}m \\ i\end{array}\right)(g-1)^{i}$. The point of considering the rational closures is the Theorem of Jennings (see [23]) which says that the converse is true: if $g-1 \in(I G)^{n}$, then $g \in G_{(n)}$. This proves Remark 1.7 (b).

\subsection{Proof of Proposition 1.4}

Let $h$ be as in the statement of Proposition 1.4. A Mayer-Vietoris argument shows that

$$
H_{1}\left(M_{h}\right) \cong H_{1}(\Sigma) / L_{-}+h_{*}\left(L_{+}\right) .
$$

Thus it suffices to show that $h_{*}\left(L_{+}\right)=L_{+} \bmod L_{-}$. First we show that $L$ has a complementary Lagrangian $L^{\prime}$ which is compatible with $i$. Assuming this then, since $h$ is symplectic, $h_{*} \mid L^{\prime}$ is the identity $\bmod L$. Thus if $\alpha \in L_{+} \cap L^{\prime}$, then $h_{*}(\alpha)-\alpha \in L \cap L_{+} \bmod L_{-}$. So, with respect to the direct sum decomposition $L_{+}=\left(L \cap L_{+}\right) \oplus\left(L^{\prime} \cap L_{+}\right), h_{*} \mid L_{+}$has the form $\left(\begin{array}{cc}I & X \\ 0 & I\end{array}\right)$.

To construct the complementary Lagrangian $L^{\prime}$ first choose any complementary summand $L_{+}^{\prime}$ of $L \cap L_{+}$in $L_{+}$. Then let $L_{-}^{\prime} \subseteq L_{-}$be the annihilator, under the intersection pairing, of $L_{+}^{\prime}$. Since $L \cap L_{-}$must pair non-singularly with $L_{+}^{\prime}$, then $L_{-}^{\prime}$ must be complementary to $L \cap L_{-}$ in $L_{-}$. It is clear that $L_{+}^{\prime}+L_{-}^{\prime}$ is the desired complementary Lagrangian.

This completes the proof of Proposition 1.4.

\subsection{Proof of Proposition 1.6}

We will first prove $\mathcal{F}_{m}^{H K} \mathcal{M}=\mathcal{F}_{m}^{K} \mathcal{M}, \mathcal{F}_{m}^{H T} \mathcal{M}=\mathcal{F}_{m}^{T} \mathcal{M}$ and $\mathcal{F}_{m}^{\mathcal{L}} \mathcal{M} \subseteq$ $\mathcal{F}_{m}^{H \mathcal{L}} \mathcal{M}$. Suppose that $i: \Sigma_{g} \subseteq M$ is an admissible surface in $M$ and $f \in \mathcal{L}_{g, 1}^{i}$. Then $i\left(\Sigma_{g}\right)$ separates $M$ into the two components $M_{+}, M_{-}$. Using handle decompositions of $M_{+}$and $M_{-}$we can find two handlebodies $H_{+} \subseteq M_{+}, H_{-} \subseteq M_{-}$such that the connected sum $\Sigma_{g^{\prime}}=i\left(\Sigma_{g}\right) \sharp \partial H_{+} \sharp \partial H_{-}$is a Heegaard embedding. Note that the complementary components of $\Sigma_{g^{\prime}}$ are $M_{ \pm}^{\prime}=\left(M_{ \pm}-H_{ \pm}\right) \sharp H_{\mp}$, using boundary connected sum. Thus we see that $M_{f}=M_{f^{\prime}}$, where $f^{\prime} \in \Gamma_{g^{\prime}, 1}$ is the image of $f$ under the canonical inclusion $\mathcal{L}_{g, 1} \subseteq \Gamma_{g, 1} \subseteq \Gamma_{g^{\prime}, 1}$. Note that if $f \in \mathcal{T}_{g, 1}$ then $f^{\prime} \in \mathcal{T}_{g^{\prime}, 1}$, and if $f \in \mathcal{K}_{g, 1}$ then $f^{\prime} \in \mathcal{K}_{g^{\prime}, 1}$. To complete the proof that $\mathcal{F}_{m}^{\mathcal{L}} \mathcal{M} \subseteq \mathcal{F}_{m}^{H} \mathcal{L} \mathcal{M}$ we will need to show that there is a compatible Lagrangian $L \subseteq H_{1}\left(\Sigma_{g^{\prime}}\right)$ so that $L \supseteq \operatorname{ker}\left\{i_{+}: H_{1}\left(\Sigma_{g}\right) \rightarrow H_{1}\left(M_{+}\right)\right\}$. (Note that $\operatorname{ker}\left\{i_{+}: H_{1}\left(\Sigma_{g}\right) \rightarrow H_{1}\left(M_{+}\right)\right\}=L_{+} \nsubseteq L_{+}^{\prime}=\operatorname{ker}\left\{i_{+}\right.$: $\left.H_{1}\left(\Sigma_{g^{\prime}}\right) \rightarrow H_{1}\left(M_{+}^{\prime}\right)\right\}$.) We show this below. If $g^{\prime}$ is large enough it follows from [20] that $M=S_{h}^{3}$ for some $h \in \mathcal{K}_{g^{\prime}, 1}$ using our chosen Heegaard surface $i_{g^{\prime}}\left(\Sigma_{g^{\prime}}\right)$. Thus $M_{f}=S_{f^{\prime} h}^{3}$. 
Now a generator of $\mathcal{F}_{m}^{\mathcal{L}} \mathcal{M}$ is, by definition, a linear combination $\sum a_{i} M_{f_{i}}$, where $\sum a_{i} f_{i} \in\left(I \mathcal{L}_{g, 1}^{i}\right)^{m}$ and all terms are defined with respect to the same embedding in $M$. But the discussion above shows that we can rewrite this as $\sum a_{i} S_{f_{i}^{\prime} h}^{3}$. Note that $\sum a_{i} f_{i}^{\prime} \in \mathbb{Q} \mathcal{L}_{g^{\prime}}^{L}$ is the image of $\sum a_{i} f_{i} \in\left(I \mathcal{L}_{g, 1}^{i}\right)^{m}$ and so belongs to $\left(I \mathcal{L}_{g^{\prime}}^{L}\right)^{m}$. Therefore $\sum a_{i} f_{i}^{\prime} h \in\left(I \mathcal{L}_{g, 1}^{L}\right)^{m}$ since $h \in \mathcal{K}_{g, 1}$. The same argument works for $\mathcal{T}_{g, 1}$ and $\mathcal{K}_{g, 1}$. Note that we have completed the proof of Proposition 1.6 for $\mathcal{F}_{m}^{K} \mathcal{M}$ and $\mathcal{F}_{m}^{T} \mathcal{M}$.

To complete the proof that $\mathcal{F}_{m}^{\mathcal{L}} \mathcal{M} \subseteq \mathcal{F}_{m}^{H \mathcal{L}} \mathcal{M}$ we need to construct the compatible Lagrangian $L \subseteq H_{1}\left(\Sigma^{\prime}\right)$. Let $A_{ \pm} \subseteq H_{1}\left(\Sigma^{\prime}\right)$ denote the subgroup generated by the boundary circles of the meridian disks of $H_{ \pm}$. Note that $A_{ \pm} \subseteq L_{\mp}^{\prime}$. We now define $L=L_{+}+A_{+}+A_{-}$. To show that this is compatible, i.e., $L=\left(L \cap L_{+}^{\prime}\right)+\left(L \cap L_{-}^{\prime}\right)$, we first observe that $L_{+} \subseteq L_{+}^{\prime}+A_{+}$. This can be seen geometrically as follows. If $\gamma$ is a closed curve in $\Sigma$ representing an element of $L_{+}$, then $i(\gamma)$ bounds a (singular) surface in $M_{+}$. This surface will intersect $H_{+}$ generically as a union of meridian disks. Thus $\gamma$-the corresponding element of $A_{+}$bounds a surface in $M_{+}^{\prime}$ and so represents an element of $L_{+}^{\prime}$. Now suppose $\alpha \in L$. Then we write $\alpha=l_{+}+h_{+}+h_{-}$, where $l_{+} \in L_{+}, h_{+} \in A_{+}, h_{-} \in A_{-}$. Now we can write $l_{+}=l_{+}^{\prime}+h_{+}^{\prime}$, where $l_{+}^{\prime} \in L_{+}^{\prime}, h_{+}^{\prime} \in A_{+}$. So now we have $\alpha=l_{+}^{\prime}+h_{+}^{\prime}+h_{+}+h_{-}$. Since $h_{+}^{\prime}+h_{+} \in L \cap L_{-}^{\prime}$ and $h_{-} \in L \cap L_{+}^{\prime}$, we have $l_{+}^{\prime} \in L$. Thus $l_{+}^{\prime} \in L \cap L_{+}^{\prime}$ and we conclude that $\alpha \in\left(L \cap L_{+}^{\prime}\right)+\left(L \cap L_{-}^{\prime}\right)$. This shows that $L$ is compatible.

This completes the proof that $\mathcal{F}_{m}^{\mathcal{L}} \mathcal{M} \subseteq \mathcal{F}_{m}^{H \mathcal{L}} \mathcal{M}$. To prove the reverse inclusion $\mathcal{F}_{m}^{H \mathcal{L}} \mathcal{M} \subseteq \mathcal{F}_{m}^{\mathcal{L}} \mathcal{M}$ we will make use of Theorem 1 which says that $\mathcal{F}_{m}^{\mathcal{L}} \mathcal{M}=\mathcal{F}_{m}^{a s} \mathcal{M}$. Thus we want to show $\mathcal{F}_{m}^{H \mathcal{L}} \mathcal{M} \subseteq \mathcal{F}_{m}^{a s} \mathcal{M}$. Since the ideal $\left(I \mathcal{L}_{g, 1}^{L}\right)^{m}$ is generated by elements of the form $\left(1-h_{1}\right) \cdots(1-$ $\left.h_{m}\right)$, where $h_{i}$ is a Dehn twist along a simple closed curve $l_{i}$ representing an element of $L$, it suffices to prove the following lemma.

Lemma 2.1. Let $l_{1}, \cdots, l_{m}$ be simple closed curves in $\Sigma$ representing elements of a Lagrangian $L$ which is compatible with an admissible embedding $i: \Sigma \subseteq M$. Let $l_{j}^{\prime}$ be translates of $i\left(l_{j}\right)$ into disjoint parallel copies of $i(\Sigma)$ in $M$. Then the link $\left\{l_{1}^{\prime}, \cdots, l_{m}^{\prime}\right\}$ is algebraically split in $M$.

Proof. Write $\left[l_{i}\right]=\lambda_{i}^{+}+\lambda_{i}^{-}$, where $\lambda_{i}^{ \pm} \in L \cap L_{ \pm}$. Then $\lambda_{i}^{ \pm}$bounds a surface $N_{i}^{ \pm}$in $M_{ \pm}$(translated). Suppose that $l_{i}^{\prime}$ lies on the $M_{+}$side of $l_{j}^{\prime}$. Then $N_{i}^{+}$is disjoint from $N_{j}^{-}$and the linking number of $l_{i}^{\prime}$ and $l_{j}^{\prime}$ 
is just the intersection number of $N_{i}^{-}$with $N_{j}^{+}$. But this is the same as the intersection number of $\lambda_{i}^{-}$with $\lambda_{j}^{+}$in $\Sigma$. Since these both represent elements of the Lagrangian $L$, the intersection number is zero. q.e.d.

This completes the proof of Proposition 1.6.

\subsection{Proof of Theorem 1(b) and Proposition 1.16}

We will need the following observation. Suppose that $M=M_{1} \cup M_{2}$, where $N=\partial M_{1}=\partial M_{2}$. Let $f, g$ be diffeomorphisms of $N$ and consider the manifold $M_{h}$ where $h=f g$. We can describe $M_{h}$ alternatively using $f$ and $g$ separately by writing $M=M_{1} \cup(I \times N) \cup M_{2}$, splitting $M$ along two parallel copies of $N \subseteq M$. Then it is easy to see that $M_{h}=M_{1} \cup_{f}(I \times N) \cup_{g} M_{2}$.

We first prove Proposition 1.16. This will follow from the preceding observation and a Theorem of Morita ([20, Proposition 2.3]) which says that any homology 3-sphere $M$ can be written in the form $S_{f}^{3}$ for some $f \in \mathcal{K}_{g, 1}$ and some $g$. Let us write $g=g_{1} \cdots g_{k}$, where each $g_{i}$ is a Dehn twist along some bounding simple closed curve $\gamma_{i}$ in the Heegaard surface $\Sigma_{g}$ in $S^{3}$. According to the observation we can split $S^{3}$ along $k$ parallel copies of $\Sigma_{g}$ in $S^{3}$ and obtain $M$ by simultaneously regluing by Dehn twists along the now disjoint copies of $\gamma_{i}$ in the parallel copies of $\Sigma_{g}$. This is the same as doing simultaneous \pm 1 surgeries along the link $L$ formed by these disjoint copies of $\gamma_{i}$. But since each $\gamma_{i}$ is a bounding simple closed curve we see that $L$ is, in fact, a boundary link." q.e.d.

We now turn to Theorem 1(b). Suppose $[M, L, f]$ is a generator of $\mathcal{F}_{m}^{b} \mathcal{M}$. The components of $L$ bound disjoint Seifert surfaces $V_{1}, \ldots, V_{m}$. Let $\Sigma$ be a connected sum of the boundaries of tubular neighborhoods of these surfaces. Thus $\Sigma$ is an admissible surface in $M$ and the components of $L$ are disjoint bounding simple closed curves $\gamma_{i}$ on $\Sigma$. Let $h_{1}, \ldots, h_{m}$ be the diffeomorphisms of $\Sigma$ defined by Dehn twists along $\gamma_{1}, \ldots, \gamma_{m}$, so that, according to Remark 1.10, cutting $M$ along $\Sigma$ and regluing using $h_{i}$ is the same as framed surgery along $\gamma_{i}$ using $f \mid \gamma_{i}$. Note that the $h_{i}$ commute with each other. If $L^{\prime}$ is any sublink of $L$, then, since the $\gamma_{i}$ are disjoint $M_{L^{\prime}, f \mid L^{\prime}}$ is obtained from $M$ by cutting along $\Sigma$ and regluing using the composition of those $h_{i}$ corresponding to the components which appear in $L^{\prime}$. Now $[M, L, f]=\sum_{L^{\prime} \subseteq L}(-1)^{\left|L^{\prime}\right|} M_{L^{\prime}, f^{\prime}}$, which is, therefore, the image, under the map $\mathcal{K}_{g, 1} \rightarrow \mathcal{M}$ of the sum

$$
\sum_{1 \leq i_{1} \leq \cdots \leq i_{k} \leq m}(-1)^{k} h_{i_{1}} \cdots h_{i_{k}}=\left(1-h_{1}\right) \cdots\left(1-h_{m}\right) .
$$


But this is obviously an element of $I \mathcal{K}_{g, 1}$.

Conversely suppose $\lambda \in\left(I \mathcal{K}_{g, 1}\right)^{m}$. Then $\lambda$ is a linear combination of elements of the form $h\left(1-g_{1}\right) \cdots\left(1-g_{m}\right)$, where $h, g_{i} \in \mathcal{K}_{g, 1}$. Now each $g_{i}$ is a product $\rho_{1} \cdots \rho_{k}$, where each $\rho_{i}$ is a Dehn twist along a bounding simple closed curve. Using the identity

$$
\rho_{1} \cdots \rho_{k}-1=\sum_{1}^{k} \rho_{1} \cdots \rho_{i-1}\left(\rho_{i}-1\right)
$$

and the normality of $\mathcal{K}_{g, 1}$ again we can assume that each $g_{i}$ is a Dehn twist along a bounding simple closed curve. Now suppose $\Sigma$ is an admissible surface in an integral homology 3 -sphere $M$. Let $\Sigma_{0}, \ldots, \Sigma_{m}$ be parallel copies of $\Sigma$ in $M$. If $g_{i}$ is a Dehn twist along $\gamma_{i} \in \Sigma$, then any $M_{h g_{i_{1}} \cdots g_{i_{k}}}$ can be obtained by cutting $M$ along $\Sigma_{0}, \Sigma_{i_{1}}, \ldots, \Sigma_{i_{k}}$ and then regluing using $h, g_{i_{1}}, \cdots, g_{i_{k}}$ on these copies of $\Sigma$. But this is the same as considering the link $L$ in $M_{h}$ defined by the $\left\{\gamma_{i} \in \Sigma_{i}\right\}$ with the framing $f$ defined by the directions of the twists given by the $\left\{g_{i}\right\}$. From this we see that the image of $h\left(1-g_{1}\right) \cdots\left(1-g_{m}\right)$ in $\mathcal{M}$ is exactly $\left[M_{h}, L, f\right]$. q.e.d.

\subsection{Proof of Theorem 1(a)}

The proof of Theorem 1(a) is very similar to that just given for (b). We need to use the result of Johnson that $\mathcal{T}_{g, 1}$ is generated by what he calls BP maps if $g \geq 3$. A BP map is obtained by doing Dehn twists along two disjoint simple closed curves in $\Sigma_{g}$, which form a bounding pair, i.e., they are homologous or, equivalently, form the boundary of a subsurface of $\Sigma_{g}$. The twists are in opposite directions. Note that the 2-component link associated to a BP map is nothing but a 1-pair blink. This gives the main motivation for the role of blinks in the present paper. Suppose we have a product $g_{1} \cdots g_{m}$ of BP maps. If we have $m$ parallel copies of an admissible surface $\Sigma$ in an integral homology 3 -sphere $M$ and, in the $i$-th copy, a pair of such curves associated to $g_{i}$, then the totality of these curves forms a blink $L_{b l}$ with a unit Seifert framing $f$ defined by the directions of the twists. Thus, just as with boundary links above, we see that the manifold obtained by cutting $M$ along $\Sigma$ and regluing by $g_{1} \cdots g_{m}$ is homeomorphic to $M_{L_{b l}, f}$. The proof of Theorem 1(a) now is identical to the proof of Theorem 1(b) with the substitution of blinks for boundary links and $\mathcal{T}_{g, 1}$ for $\mathcal{K}_{g, 1}$. 


\subsection{Proof of Theorem 1(c)}

Suppose $[M, L, f]$ is a generator of $\mathcal{F}_{m}^{a s} \mathcal{M}$. Consider a tubular neighborhood of $L$ and define a connected submanifold $M_{1} \subseteq M$ by connecting these components by solid tubes in $M$. For each component $l_{i}$ of $L$ there is a canonical longitude $\lambda_{i} \subseteq \partial M_{1}$ defined by the requirement that $\lambda_{i}$ be null-homologous in the complement of $l_{i}$. Since $L$ is algebraically split. $\left\{\lambda_{i}\right\}$ span $\operatorname{Ker}\left\{H_{1}\left(\partial M_{1}\right) \rightarrow H_{1}\left(\overline{M-M_{1}}\right)\right\}$ which is a Lagrangian in $H_{1}\left(\partial M_{1}\right)$. The result of doing \pm 1 -surgery on any sublink of $L$ is the same as cutting $M$ along $\partial M_{1}$ and regluing by simultaneous Dehn twists on the corresponding $\lambda_{i} \subseteq \partial M_{1}$. If we choose an identification $\Sigma_{g} \cong \partial M_{1}$, these Dehn twists define elements $h_{i} \in \mathcal{L}_{g .1}^{i}$, where $i$ is the composition $\Sigma_{g} \cong \partial M_{1} \subseteq M$. As in Section 2.4 we see that $[M, L, f]$ is the image, under the map $\mathcal{L}_{g, 1} \rightarrow \mathcal{M}$ defined by $i$, of $\left(1-h_{1}\right) \cdots\left(1-h_{m}\right)$.

We now prove the converse statement. Suppose $\lambda \in\left(I \mathcal{L}_{g, 1}\right)^{m}$. Then $\lambda$ is a linear combination of elements of the form $h\left(1-g_{1}\right) \cdots\left(1-g_{m}\right)$. where $h, g_{i} \in \mathcal{L}_{g, 1}$. Now writing each $g_{i}$ as a product of $L$-twists and using repeatedly the identity $1-g h=g(1-h)+1-g$, we can write this as a sum of elements of the form $h\left(1-\rho_{1}\right) \cdots\left(1-\rho_{m}\right)$, where each $\rho_{i}$ is an $L$ - twist.

We now proceed as in Sections 2.4 and 2.5. We see that the image of $h\left(1-\rho_{1}\right) \cdots\left(1-\rho_{m}\right)$ in $\mathcal{M}$ is an element $[M, K, f]$ which can be described as follows. We begin with an embedded surface $\Sigma$ in some integral homology 3-sphere $M^{\prime}$ and let $M_{1}$ be the closure of one of the components of $M^{\prime}-\Sigma$, so that $L=\operatorname{ker}\left\{H_{1}(\Sigma) \rightarrow H_{1}\left(M_{1}\right)\right\}$. Let $\Sigma, \Sigma_{1}, \cdots, \Sigma_{m}$ be parallel copies of $\Sigma$ appearing in that order as we move away from $M_{1}$. Then $\rho_{i}$ is a Dehn twist on a curve $\lambda_{i} \subseteq \Sigma_{i}$, and $\lambda_{i}$ is null-homologous in $M_{1}$. Thus $M$ is obtained by cutting $M^{\prime}$ along $\Sigma$ and reattaching with $h, K$ is the link consisting of the $\left\{\lambda_{i}\right\}$ and $f$ is the framing defined by the signs of the Dehn twists. To complete the proof we need to see that $K$ is algebraically split. But since $\lambda_{i}$ bounds a chain in $M_{1}$ and $h$ does not alter this, it follows that the linking number of $\lambda_{i}$ with any $\lambda_{j}$, when $j>i$, is zero.

\section{Equivalence of $A S, B$ and $B L$ filtrations}

In this section we prove Theorems 2,3 and 4. 


\subsection{A review of relations on $\mathcal{M}$ in graphical and algebraic form}

In this section we review the forms of notation used in this paper as well as some important identities among the elements of $\mathcal{M}$. We follow the conventions of [22], [5], [8]. For the convenience of the uninitiated reader, we review them again here. The notation comes in two forms: algebraic and graphical. By algebraic notation we mean $[M, L, f]$. Note that the various filtrations on $\mathcal{M}$ have been written in algebraic notation. The rules of the graphical notation are summarized in Figure 3. Since we are talking about identities of (linear combinations) of integral homology 3spheres obtained by framed links in other integral homology 3-spheres, it is almost unavoidable to use graphical notation to represent framed links, and surgeries on them.

Remark 3.1. We mention once and for all that any link (whether algebraically split or boundary or blink) drawn in a figure corresponds to a linear combination of integral homology 3-spheres, and therefore represents an element of $\mathcal{M}$. The figures represent identities of these elements. We cannot stress too strongly the fact that in papers prior to the ones talking about finite type invariants figures corresponded to links or 3-manifolds, but never to linear combinations of them. Nevertheless, this point of view is very fundamental in the world of finite type invariants.
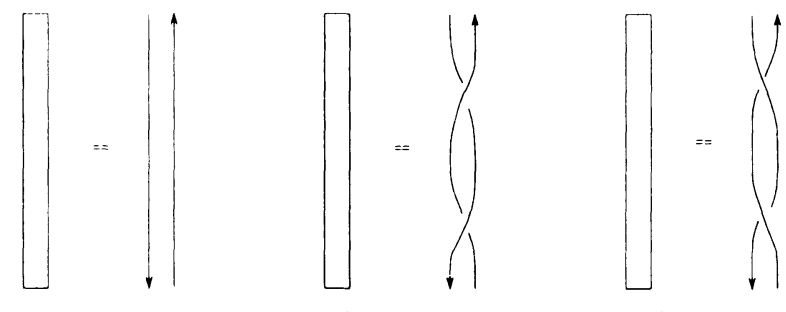

Figure 3. Some drawing conventions for bands. Shown here are ribbon parts of $A S$-admissible links that represent (linear combinations of) integral homology 3-spheres. The numbers in the bottom of each band indicate the number of twists that we put in the band.

We are now ready to review identities of elements of $\mathcal{M}$. We begin with the following fundamental identity, (in algebraic notation) for an $A S$-admissible link $(L, f)$ in an integral homology 3-sphere $M$ : 
Let $L_{b l} \cup L$ be the union of an $A S$-admissible link $L$ and a $B L$ admissible blink $L_{b l}$ (in the complement of $L$ ). Let $l$ denote either a component of $L$, or a 1-pair of $L_{b l}$. Define

$$
L^{\prime}= \begin{cases}L & \text { if } l \notin L, \\ L-l & \text { if } l \in L,\end{cases}
$$

and

$$
L_{b l}^{\prime}= \begin{cases}L_{b l} & \text { if } l \notin L_{b l}, \\ L_{b l}-l & \text { if } l \in L_{b l} .\end{cases}
$$

Let $f^{\prime}$ (respectively, $\left.f\right|_{l}$ ) denote the restriction of the framing $f$ of $L_{b l} \cup L$ to $L_{b l}^{\prime} \cup L^{\prime}$ (respectively $l$ ). Then we have the following fundamental relation:

$$
\left[M, L_{b l} \cup L, f\right]=\left[M, L_{b l}^{\prime} \cup L^{\prime}, f^{\prime}\right]-\left[M_{\left(l,\left.f\right|_{l}\right)} L_{b l}^{\prime} \cup L^{\prime}, f^{\prime}\right] .
$$

The proof of the above equation follows by definition of the symbol $\left[M, L_{b l} \cup L, f\right]$ and the following exercise, left to the reader:

Exercise 3.2. Show that the unit-Seifert framing $f \mid L_{b l}$ of $L_{b l}$ in $M$ is the same as the one of $L_{b l}$ in $M_{\left(l,\left.f\right|_{l}\right)}$.

Remark 3.3. This extends the notation of previous papers [3], [5], [6], [8], [9], where $L_{b l}$ is the empty blink. If $l$ is a knot that bounds a disk $D$ in $M$, then $M_{\left(l,\left.f\right|_{l}\right)}$ is diffeomorphic to $M$ and we may construct the link in $M$ corresponding to $L_{b l}^{\prime} \cup L^{\prime}$ in $M_{\left(l,\left.f\right|_{l}\right)}$ from $L_{b l}^{\prime} \cup L^{\prime}$ by just giving the bundle of strands of $L_{b l}^{\prime} \cup L^{\prime}$ which pass through $D$ a full clockwise twist if $f=+1$ or counterclockwise twist if $f=-1$.

Examples of equation (13) in graphical notation are given in Figures 4,5 and 6.

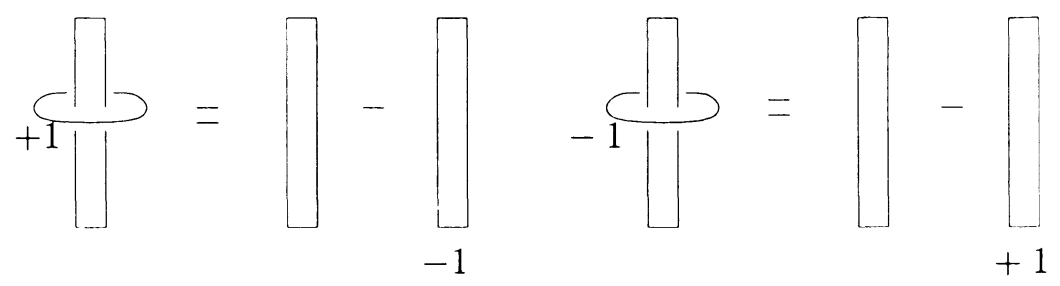

Figure 4. A special case of equation (13) in a graphical way. 

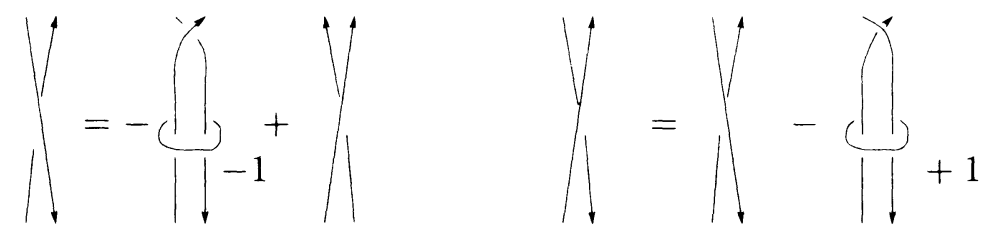

Figure 5. Another special case of equation (13). The figure represents an identity in $\mathcal{M}$. There are two interpretations of the above figure. Either each of the crossings shown belongs to the same component (of an algebraically split link), or each of the crossings shown is part of a ribbon of a piece of a Seifert surface of a 1-pair blink. It will be clear each time we use the identity shown in the figure which interpretation we have in mind.

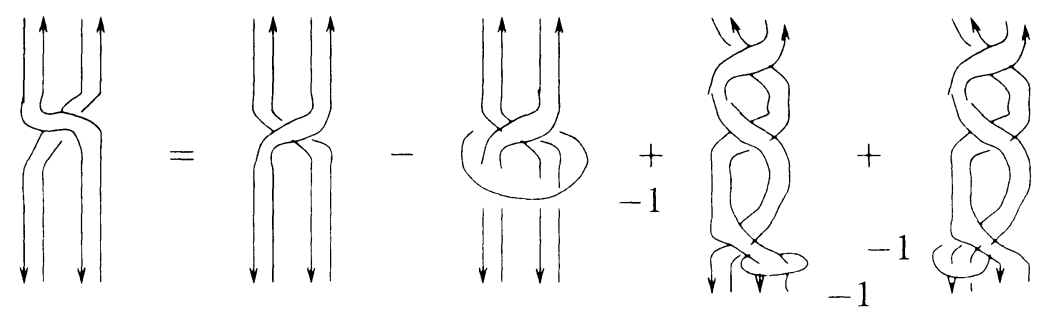

Figure 6. Another special case of equation (13). The figure represents an identity in $\mathcal{M}$. Crossings are in the same component of the link. 


\subsection{A 4-term relation on $\mathcal{M}$}

In this and in the two sections we introduce three versions of a 4-term relation on $\mathcal{M}$, which will be used crucially in the proof of Theorem 3 .

We begin by noting that there is a map from +1 -framed knots in $S^{3}$ to $\mathcal{M}$, defined by $K \rightarrow\left[K, S^{3},+1\right]=S^{3}-S_{K,+1}^{3}$ originally introduced in [3]. Dually this map induces a map from invariants of integral homology 3 -spheres to invariants of knots in $S^{3}$. Furthermore it is trivial to show that finite type invariants of integral homology 3-spheres map to finite type invariants of knots. Actually much more is known, namely that $A S$-type $3 m$ of integral homology 3 -spheres map to type $2 m$ invariants of knots; see [10] and [6]. The vector space on the set of knots satisfies a basic relation, the 4 -term relation. This relation plays an important role in studying finite type invariants of knots. According to the above defined map, we have a 4-term relation satisfied on the image of this map in $\mathcal{M}$. Strangely enough, the above relation has not been explicitly introduced or noticed before. In the present paper we describe the 4term relation on $\mathcal{M}$ and use it in a crucial way in the proof of Theorem 3. Furthermore, in Section 1.7 we pose the question of a possible relation between the 4-term relation and the more well known $A S$ and $I H X$ relations on $\mathcal{M}$. With these preliminaries and motivation in mind, we begin to describe the 4-term relation on $\mathcal{M}$.

Let $C \cup K$ be a two-component sublink of an $A S$-admissible link $L$ in $S^{3}$, such that the following hold:

- The intersection of $K$ with a 3 -ball $B$ in $S^{3}$ consists of 3 arcs shown in Figure 10.

- $C$ is an unknot that bounds a disc $D$ which lies in the interior of the ball $B$. The disc $D$ intersects $K$ in two points, $b, c$. See Figure 10.

Choose 4 disjoint discs $D_{j}$ (for $\left.j=s, n, e, w\right)(s, n, e, w$ stands for south, north, east and west) in the ball that intersect $K$ in the 4 tuples of points $(j, a)$, as shown in Figure 12 with the abbreviations of Figure 11. Let $C_{j}$ (for $j=s, n, e, w$ ) denote the boundary of $D_{j}$ with framing -1 . Note that $C_{j} \cup L$ is an $A S$-admissible link in $S^{3}$. Let us momentarily abbreviate the elements $\left[S^{3}, C_{j} \cup L,-1 \cup f\right]$ by $\left[C_{j} \cup L\right]$, where $f$ is the framing of the $A S$-admissible link $L$. We can now state the following proposition: 

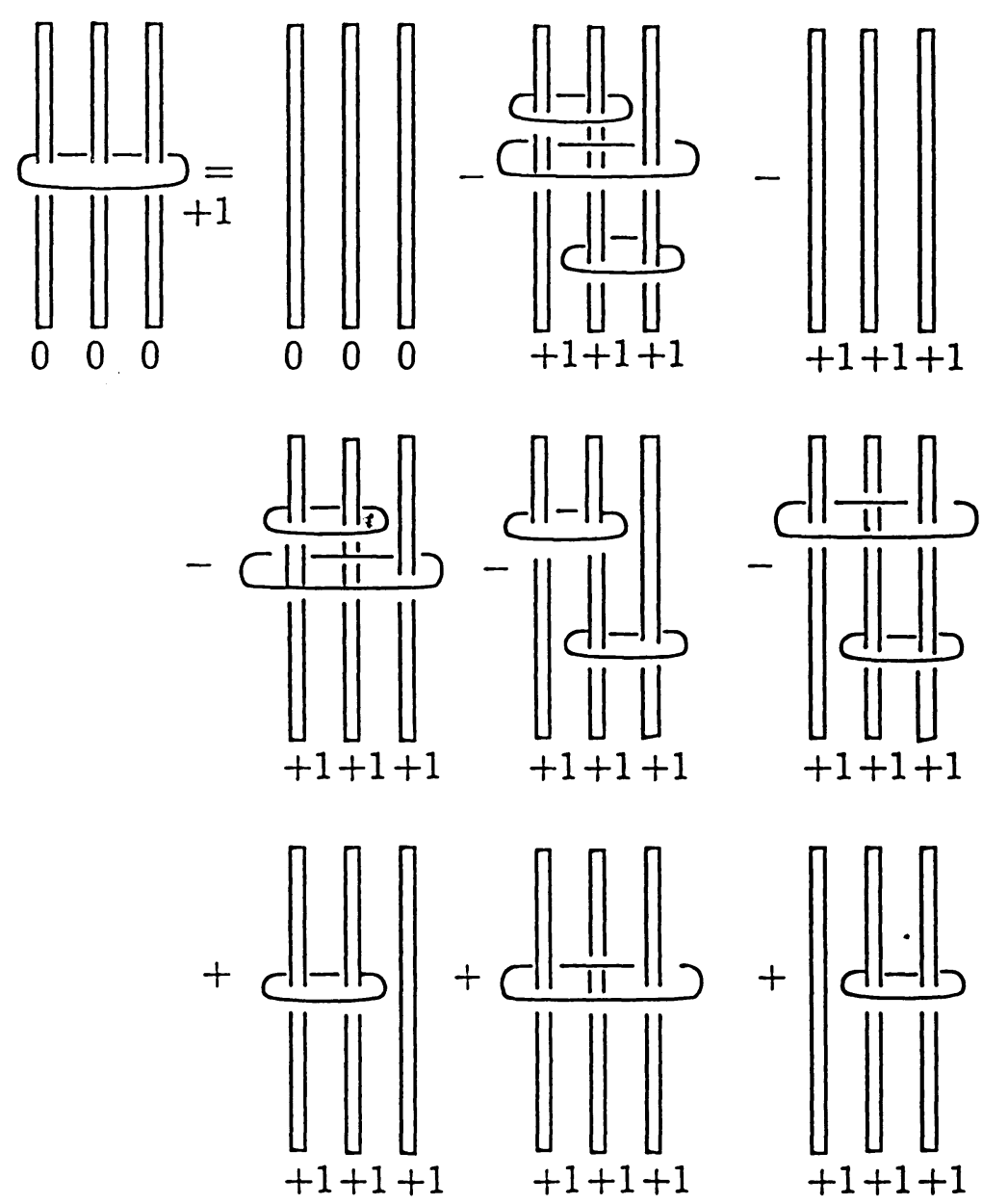

Figure 7. A figure showing an identity in $\mathcal{M}$. The links shown are algebraically split. For a proof, see [5]. The numbers in the bottom of each band represent twists, with the conventions of Figure 3 . The framings in all the horizontal components are +1 . 


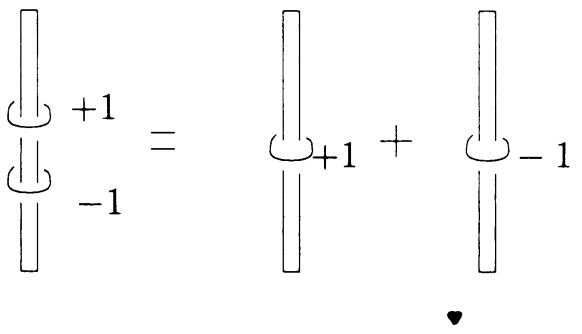

Figure 8. A figure showing an identity in $\mathcal{M}$. The links shown are algebraically split. For a proof, see [5]. This figure is used to change the +1 framing of an unknot to a -1 and vice versa.

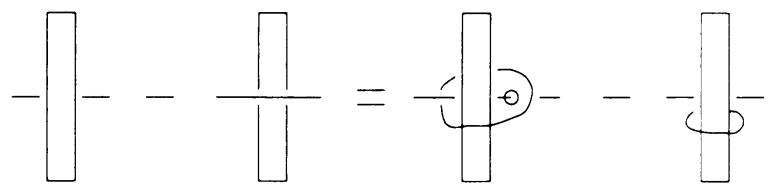

Figure 9. Another special case of equation (13). We assume that all the arcs in the band lie in the same link component. Then the link shown in the third picture from the left contains a unit Seifert-framed 1-pair blink (indeed, it bounds a Seifert surface obtained by tubing the obvious disc along the strands contained in the band). The fourth picture from the left contains a 1-component boundary link in the complement of the rest of the link, and thus (using Remark 1.13) a 1-pair blink.

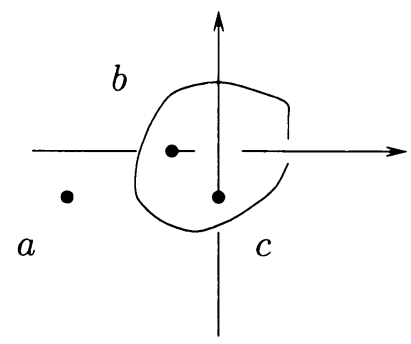

Figure 10. We show the intersection of a 3-ball $B$ with a knot $K$, which consists of 3 arcs (two of them are shown, the third is perpendicular to the page pointing towards you). Shown also are 3 points $a, b, c$ on the knot $K$, as well as an unknot that bounds a disc which intersects $K$ in $b, c$ 
Proposition 3.4. With the above notation, we have the following relation on $\mathcal{M}$ :

$$
\left[C_{s} \cup L\right]-\left[C_{n} \cup L\right]=\left[C_{e} \cup L\right]-\left[C_{w} \cup L\right] .
$$

We call the above relation the (first version of the) 4-term relation on $\mathcal{M}$.

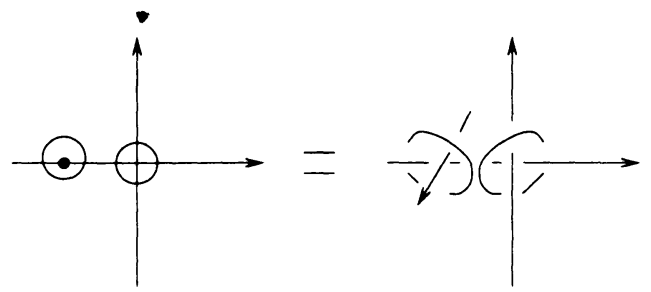

FIgURE 11. Some abbreviation conventions for drawing the next figure.
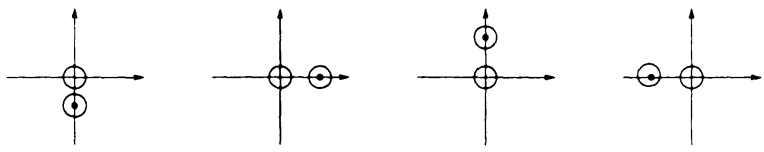

Figure 12. The terms $s, e, n, w$ appearing in the 4 -term relation on $\mathcal{M}$ with the drawing conventions of the previous figure.

Proof. The proof of the 4-term relation on $\mathcal{M}$ is the same as the proof of the 4-term relation on the space of knots; see [1]. In both proofs, we move the arc $a$ of Figure 10 from the $S W$ quarter, to the $N E$ quarter in two ways: by passing through either the $N W$ quarter, or the $S E$ quarter. Using Figure 13, the difference in the first (respectively, second) way equals to the left (respectively, right)-hand side of equation (14), thus proving the proposition. q.e.d.

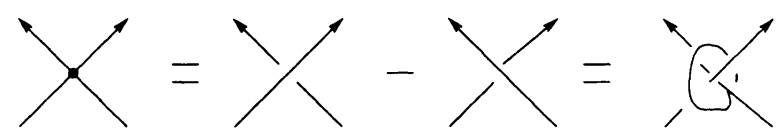

FIgURE 13. An identity useful for the 4-term relation.

Remark 3.5. The points $a, b, c, s, w, e, n$ (on the knot $K$ ) are displayed in the order shown in Figure 14. 


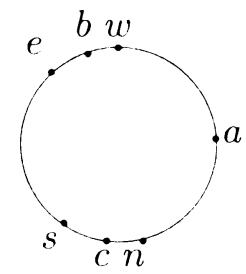

Figure 14. The order various points on a knot $K$.

Remark 3.6. If we represent the knot $K$ by a circle and the knots $C, C_{j}$ (for $j=s, n, e, w$ ) by chords (that intersect the above mentioned circle in two points each, namely the points of intersection $K \cap D_{j}$ ), then the 4-term relation reads as in Figure 15. The 4-term relation will be used, in the form of Figure 15, in the proof of Theorem 3.

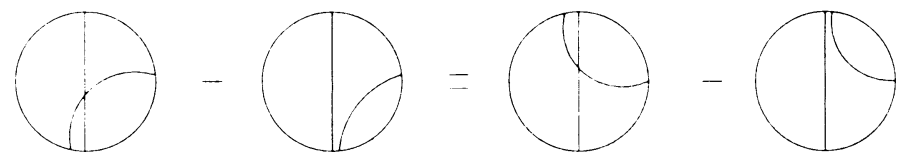

Figure 15. The first version of the 4-term relation on $\mathcal{M}$, with the notation of Remark 3.6.

\subsection{Two more versions of the 4 -term relation on $\mathcal{M}$}

In this section we will introduce two more refined versions of the 4-term relation on $\mathcal{M}$.

Let $C \cup K_{1} \cup K_{2}$ be a three-component sublink of an $A S$-admissible link $L$ in $S^{3}$, such that there is a three-ball $B$ in $S^{3}$ with the following properties:

- The intersection of $K_{1}$ with $B$ consists of 3 arcs shown in Figure 16.

- The intersection of $K_{2}$ with $B$ consists of 2 arcs shown in Figure 16.

- $C$ is an unknot that bounds a disc $D$ which lies in the interior of the ball $B$. The disc $D$ intersects $K_{1}$ in two points and intersects $K_{2}$ in two points. Furthermore, $D$ intersects no other component of $L$. See Figure 16 . 
Let $D_{j}$ (for $j=s, n, e, w$ ) be 4 discs as in Section 3.2 (with Figure 16 replacing Figure 10). Let $C_{j}$ be the boundary of the disc $D_{j}$.

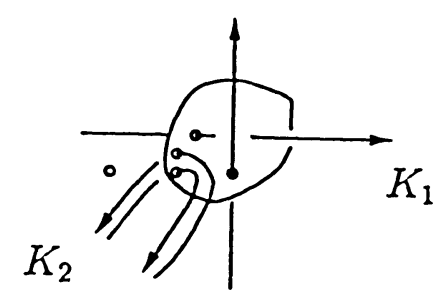

Figure 16. Shown here are the 3 arcs along which a ball $B$ intersects the knot $K_{1}$ and the 2 arcs along which it intersects the knot $K_{2}$. Shown also with bold are points of $K_{1}$ and $K_{2}$ where they intersect $D$.

Proposition 3.7. With the above notation, we have the following relation on $\mathcal{M}$ :

$$
\left[C_{s} \cup L\right]-\left[C_{n} \cup L\right]=\left[C_{e} \cup L\right]-\left[C_{w} \cup L\right]+\left[E_{1}^{\prime}\right]-\left[E_{2}^{\prime}\right],
$$

where $E_{1}, E_{2}$ are links shown in Figure 17. In the rest of the paper, the above relation will be called the (second version of the) 4-term relation on $\mathcal{M}$.
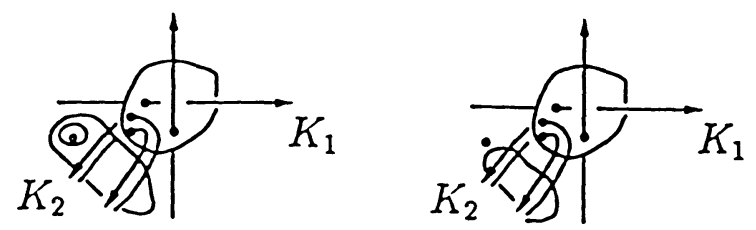

Figure 17. Shown here are the two links $E_{1}^{\prime}$ and $E_{2}^{\prime}$ mentioned in equation (15). Note that $E_{1}^{\prime}$ contains a 1-pair blink and $E_{2}^{\prime}$ contains a 1-component boundary link.

Proof. Moving the arc of $K$ (of Figure 16) from the $S W$ quarter to the $N E$ quarter as in Proposition 3.4, it follows that:

$$
\left[C_{s} \cup L\right]-\left[C_{n} \cup L\right]-\left[C_{e} \cup L\right]+\left[C_{w} \cup L\right]=\left[E_{1}\right]-\left[E_{2}\right],
$$

where $E_{1}, E_{2}$ are as in Figure 18. Using Figure 9 the result follows. q.e.d. 

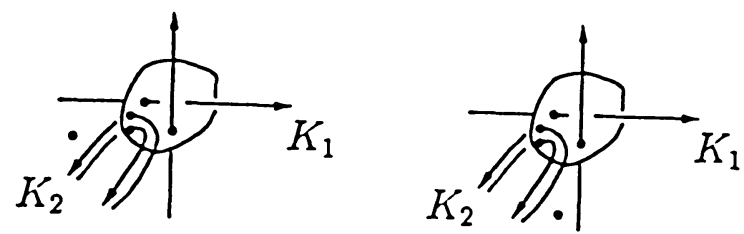

Figure 18. Shown here are the links $E_{1}$ and $E_{2}$ of equation (16).

A few remarks are in order:

Remark 3.8. In analogy with Remark 3.6, if we represent the knots $K_{1}, K_{2}$ by two circles and the knots $C, C_{j}$ (for $j=s, n, e, w$ ) by chords (that intersect the above mentioned circles in two points each, namely the points of intersection $K_{1} \cap D_{j}$ and $K_{2} \cap D_{j}$ ) then the 4-term relation reads as in Figure 19. Note also that the error terms contain 1-pair blinks in the complement of the link $L-C$. The 4-term relation will be used, in the form of Figure 19, in the proof of Theorem 3.

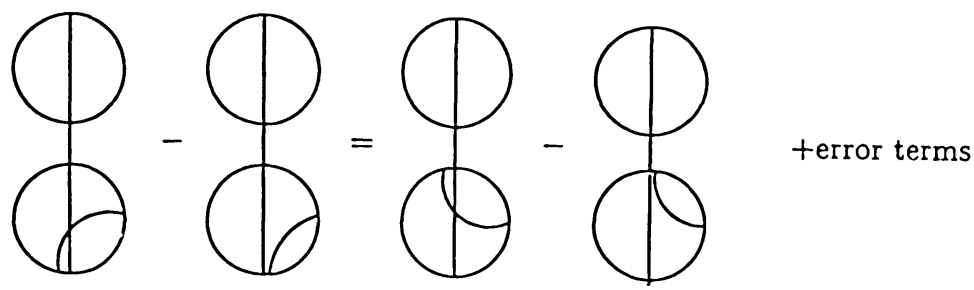

Figure 19. The second version of the 4-term relation on $\mathcal{M}$, with the notation of Remark 3.8 .

Remark 3.9. Proposition 3.7 implies Proposition 3.4. Indeed, consider Figure 20. Notice that in this case, the error terms vanish. The reason that we introduced Proposition 3.4 at all was as a warm-up exercise to make the proof of Proposition 3.7 more accessible, in light of the similarity between Proposition 3.4 and the 4 -term relation in the theory of finite type knot invariants; see [1]. 


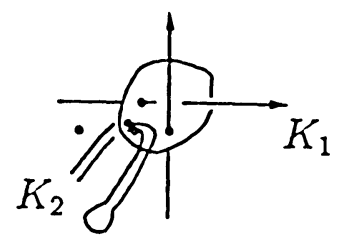

Figure 20. A special link that reduces Proposition 3.7 to Proposition 3.4.
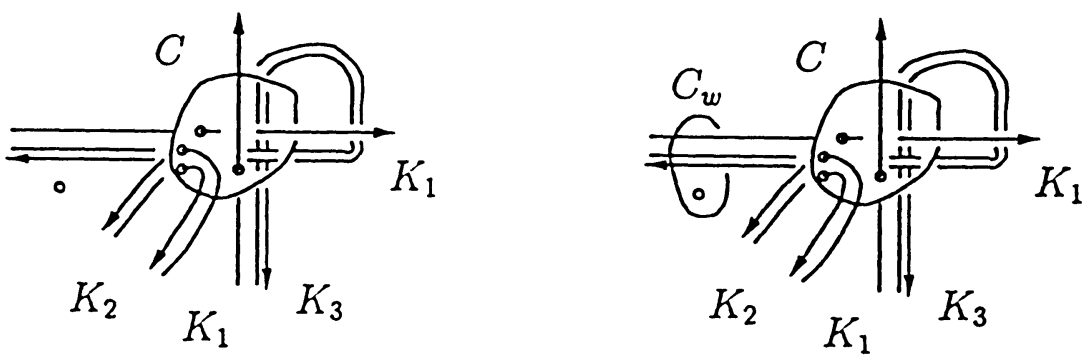

Figure 21. Shown here on the left-hand side are the 3 arcs along which a ball $B$ intersects the knot $K_{1}$, the 2 arcs along which it intersects $K_{2}$ and the 2 arcs that it intersects the knot $K_{3}$. Shown also is an unknotted component $C$ which bounds a disc $D$, and with bold dots are the points where $D$ intersects $K_{1}$ and $K_{2}$. On the right-hand side of the figure is shown one of the links $\left(C_{w}\right)$ that appears in equation (17). 
We close this section with the third (and most refined) version of the 4-term relation on $\mathcal{M}$.

Proposition 3.10. Let $C \cup K_{1} \cup K_{2} \cup K_{3}$ be a four-component sublink of an $A S$-admissible link $L$ in $S^{3}$. Assume that there is a ball $B$ such that the intersection of $L$ with $B$ is as in Figure 21. Let $D$ and $D_{j}$ (for $j=s, n, e, w$ ) be discs as in Section 3.2 (with Figure 21 replacing Figure 10). Let $C_{j}$ be the boundary of the disc $D_{j}$ and $C$ the boundary of $D$. Then, with the abbreviations before the statement of Proposition 3.4, we have the following:

$$
\left[C_{s} \cup L\right]-\left[C_{n} \cup L\right]=\left[C_{e} \cup L\right]-\left[C_{w} \cup L\right]+\text { error terms, }
$$

where the error terms include 1-pair blinks in the complement of $L-\left(C \cup_{j} C_{j}\right)$. In the rest of the paper, the above relation will be called the (third version of the) 4-term relation on $\mathcal{M}$.

Proof. The proof is similar to that of Proposition 3.7 and we briefly sketch it here. Using the equation of Figure 22, after moving the arc of $K$ (of Figure 21) from the $S W$ quarter to the $N E$ quarter, we get the following equality involving the terms $\left[C_{j} \cup L\right]$, as well as two kinds of error terms: $E T_{1}$ (respectively, $E T_{2}$ ) that come from moving the $K_{1}$ arc around the $K_{3}$ arcs (respectively, around the $K_{2}$ arcs):

$$
\left[C_{s} \cup L\right]-\left[C_{n} \cup L\right]=\left[C_{e} \cup L\right]-\left[C_{w} \cup L\right]+E T_{1}+E T_{2} .
$$

By definition, we have:

$$
E T_{1}=\sum_{i=1,2}\left(\left[E_{i, s}\right]-\left[E_{i, n}\right]\right)-\left(\left[E_{i, e}\right]-\left[E_{i, w}\right]\right),
$$

where $E_{i, j}$ (for $i=1,2$ and $j=s, n, e, w$ ) are 1-pair blinks that come from Figure 22. Each of the differences in the parentheses is a sum (with signs) of four terms (recall that for a 1-pair blink $E,[E]$ is a sum (with signs) of two terms), two of which cancel. The remaining two (with signs) can be combined as $[e]$ for some 1-pair blink $e$. These 1pair blinks $\{e\}$ bound Seifert surfaces in the interior of the ball $B$ (of Figure 21), and these surfaces intersect the disc that $C$ bounds, where $C$ is as in Figure 21. By the same argument as in Proposition 3.7, it follows that $E T_{2}$ can be written as a linear combination of links that contain 1-pair blinks that bound surfaces in the interior of the ball $B$. This proves Proposition 3.10. q.e.d. 


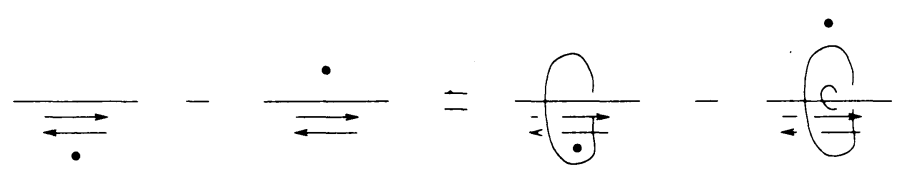

Figure 22. Moving an arc perpendicular to the page past three others. On the left-hand side, there are four arcs shown, one perpendicular to the plane, pointing towards your eyes. Of these four arcs, the top horizontal one and the one represented by a dot, belong to the same link component, and so do the two others. Note that only two components of the link are involved, and that this figure is a redrawing of Figure 9. The two blinks on the right-hand side are denoted by $E_{1}$ and $E_{2}$ respectively.

Remark 3.11. In analogy with Remark 3.8, if we represent the knots $K_{1}, K_{2}, K_{3}$ by three circles and the knots $C, C_{j}$ (for $\left.j=s, n, e, w\right)$ by chords (that intersect the above mentioned circles in two points each, namely the points of intersection $K_{i} \cap D_{j}$ (for $i=1,2,3$ ), then the 4term relation reads as in Figure 23. Note that the error terms contain 1-pair blinks in the complement of the link $L-\left(C \cup_{j} C_{j}\right)$. The 4-term relation will be used, in the form of Figure 23, in the proof of Theorem 3.

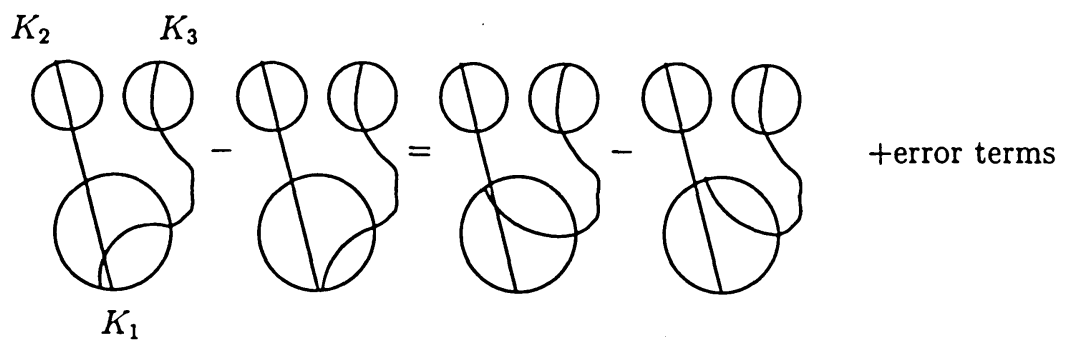

Figure 23. The third version of the 4-term relation on $\mathcal{M}$, with the notation of Remark 3.11. 


\subsection{Proof of Theorem 3}

This section is devoted to the proof of Theorem 3. The proof is long, and rather involved. For the convenience of the reader, we divide the proof in three propositions, each of which needs independent arguments.

All links considered in this section are $A S$-admissible links in $S^{3}$. Let us begin by introducing a definition that is useful in this section.

Definition 3.12. An $A S$-admissible link $(L, f)$ (in $S^{3}$ ) is near to another one $\left(L^{\prime}, f^{\prime}\right)$ (of not necessarily the same number of components) if there is a finite set of $A S$-admissible $L^{\prime \prime}$ containing $L^{\prime}$ such that:

$$
\left[S^{3}, L, f\right]=\sum_{L^{\prime \prime}}\left[S^{3}, L^{\prime \prime}, f^{\prime \prime}\right] \in \mathcal{F}_{*}^{a s} \mathcal{M}
$$

We caution the reader that being "near to" is not a symmetric relation. Note also that any link is near to an arbitrary sublink of it. A note on transitivity of the relation "near to": if $(L, f)$ is near to $\left(L^{\prime}, f^{\prime}\right)$, which itself is near to a third one $\left(L^{\prime \prime}, f^{\prime \prime}\right)$, then it is not clear that $(L, f)$ is near to $\left(L^{\prime \prime}, f^{\prime \prime}\right)$. However, if $\left(L^{\prime \prime}, f^{\prime \prime}\right)$ is obtained from $\left(L^{\prime}, f^{\prime}\right)$ (and $\left(L^{\prime}, f^{\prime}\right)$ is obtained from $\left.(L, f)\right)$ using the equalities of Figures 5,6 and 7 , then transitivity holds. We will use transitivity freely in the proof of Proposition 3.13, because of the above note. As a variation, we call a finite linear combination of $A S$-admissible links $\left(L_{i}, f_{i}\right)$ near to an $A S$-admissible link $\left(L^{\prime}, f^{\prime}\right)$ if there is a finite number of $A S$-admissible links $\left(L^{\prime \prime}, f^{\prime \prime}\right)$ that include $\left(L^{\prime}, f^{\prime}\right)$ such that:

$$
\sum_{i}\left[S^{3}, L_{i}, f_{i}\right]=\sum_{L^{\prime \prime}}\left[S^{3}, L^{\prime \prime}, f^{\prime \prime}\right] \in \mathcal{F}_{*}^{a s} \mathcal{M}
$$

With the above terminology we have the following:

Proposition 3.13. For every AS-admissible $4 m$-component link in $S^{3}$ there is a trivial $L_{t r}(m) m$-component link such that $(L, f)$ is near to $\left(L_{t r}(m), f(m)\right)$.

Proof of proposition 3.13. We begin by remarking that the statement in Proposition 3.13 is a finiteness statement, and not one using downward induction. Furthermor, using Figure 8 it follows that if the above proposition holds for one choice of unit framings, then it holds for all. We will therefore omit mentioning the framings in the proof given below.

We divide the proof of the proposition in two steps. 

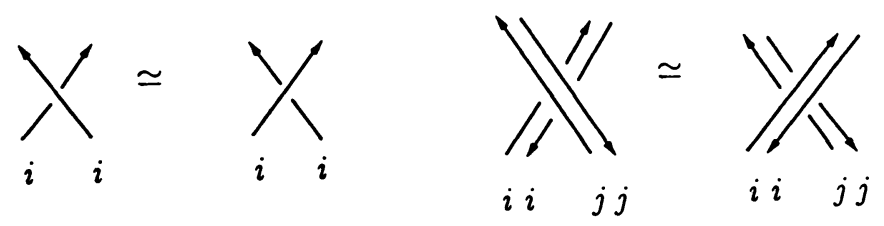

Figure 24. Two local moves that generate the relation of surgery equivalence. Here arcs labeled by the same letter ( $i$ or j) belong to the same link component.

- Step $17 L$ is near to a (finite linear combination of) $L(\Gamma)$ where $L(\Gamma)$ are links obtained by trivalent vertex oriented graphs with $4 m$ edges as in [3], [5].

Proof. The proof is similar to the proof of [6, Theorem 4]. Note that the theorem $\left[6\right.$, Theorem 4] states that $\left[S^{3}, L, f\right] \in \mathcal{G}_{4 m}^{a s} \mathcal{M}$ can be written as a linear combination of terms of the form $\left[S^{3}, L(\Gamma), f\right]$ where $\Gamma$ are trivalent vertex oriented graphs of $4 m$ edges. In that theorem, we first alter $L$ by a surgery equivalent one (where surgery equivalence is the relation generated by the local moves of Figure 24). Using a relation shown in graphical form in Figure 7 we then replace $L$ by a linear combination of $L(\Gamma)$ for trivalent vertex oriented graphs of $4 m$ edges.

Our present claim (of step 1) follows from the same argument (sketched above) that shows [6, Theorem 4], after we use the equations (shown in graphical notation) in Figures 5, 6 and 7. Note that in Figures 5,6 and 7 the extra terms that are present give links that contain the links obtained by trivalent graphs. q.e.d.

- Step 2 If $L(\Gamma)$ is a link obtained by a trivalent graph of $4 m$ edges, then $L(\Gamma)$ is near a trivial $m$-component link $L_{t r}(m)$.

Proof. This essentially follows from Lemma 3.4 of [3]. For completeness, we repeat the argument here. Take a forest Forest of $\Gamma$ containing all of the vertices of $\Gamma$, and consider $L$ (Forest). This is a sublink of $L(\Gamma)$, therefore $L(\Gamma)$ is near to $L($ Forest $)$, and an Euler characteristic argument shows that $L$ (Forest) has at least $m$ components. q.e.d. 
The proof of Proposition 3.13 is complete. q.e.d.

We also have the following proposition, that depends crucially on the existence of the 4-term relation shown in equation (14).

Proposition 3.14. There is a positive constant $c \in \mathbb{N}$ such that for every $A S$-admissible link $(L, f)$ in $S^{3}$ that contains a sublink $L_{t r}\left(\mathrm{~cm}^{3}\right) \cup$ $K$ with the following properties:

- $L_{t r}\left(\mathrm{~cm}^{3}\right)$ is a trivial link of $\mathrm{cm}^{3}$ components which bounds a disjoint union of discs $\cup_{i} D_{i}$.

- Each disc $D_{i}$ intersects the knot $K$ in two points, and intersects no other component of $L$.

Hence we have that $\left[S^{3}, L, f\right] \in \mathcal{F}_{m}^{b} \mathcal{M}$, so (using Remark 1.13) that $\left[S^{3}, L, f\right] \in \mathcal{F}_{m}^{b l} \mathcal{M}$ as well.

Before we give the proof of it, let us introduce one more definition that will be useful in stating the proof. Recall (see e.g. [1]) the combinatorial notion of a chord diagram with support on a circle.

Definition 3.15. A chord diagram is called $m$-boundary if it contains $m$ nonintersecting chords. For an example, see Figure 25.
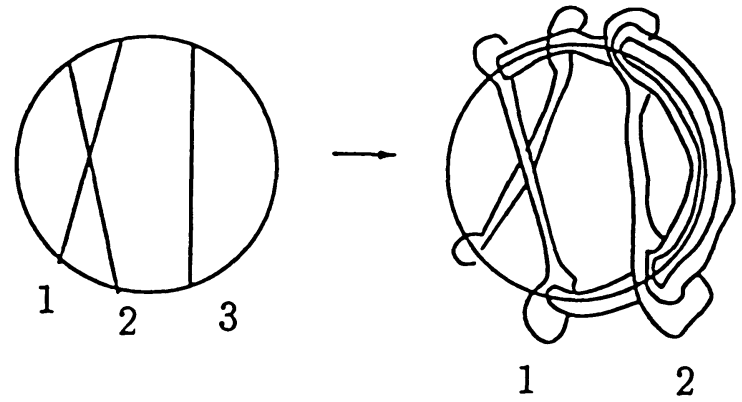

Figure 25. Shown on the left is a chord diagram with 3 chords. Two of them (1 and 3$)$ do not intersect thus the chord diagram is 2- boundary. On the right is shown the result of tubing it along the two nonintersecting chords. The result is a boundary link of two components in the complement of a two component link.

Proof. Let $L$ and $K$ be as in the statement of Proposition 3.14, and let $C D_{K}$ denote the associated chord diagram of $K$, .i.e., the ordered 
set of points of the intersection of $K$ with the disk $D_{i}$. The two points of intersection of $D_{i} \cap K$ will be represented by a chord of $C D_{K}$, as in the theory of finite type invariants of knots.

We now give the proof in six steps:

- Step 1 If $C D_{K}$ is $m$-boundary, then $\left[S^{3}, L, f\right] \in \mathcal{F}_{m}^{b} \mathcal{M}$.

Proof. Indeed, tube the $m$ discs that represent $m$ nonintersecting chords, using an innermost circle argument. For an example, see Figure 25. The result is a boundary $m$ component sublink $L_{b}$ of $L$ that bounds surfaces in the complement of $L-L_{b}$, from which it follows easily that $\left[S^{3}, L, f\right] \in \mathcal{F}_{m}^{b} \mathcal{M}$. Note that the result is independent of the fact that $K$ may be knotted; it only depends on the associated chord diagram of $K$. q.e.d.

Now, if $C D_{K}$ is not an $m$-boundary chord diagram, we have the following steps.

- Step 2 We can always assume that the framing in each of the components of $L_{t r}\left(\mathrm{~cm}^{4}\right)$ is +1 or -1 as we please.

Proof. Use the equation in Figure 8. q.e.d.

Before we state the next step, let us introduce some useful terminology. We say that a chord diagram is represented by an $m$-tower if it can be written using the 4-term relation (see Figure 15) as a (finite) linear combination of $m$-boundary chord diagrams. With this terminology we have the next step:

- Step 3 If a chord diagram contains at least $\mathrm{cm}^{3}$ chords (where $c>1$ fixed positive integer), and is represented by an $m-1$ tower, then it is represented by an $m$-tower.

Proof. Without loss of generality, we may assume that the chord diagram contains an $m-1$ tower, $T_{m-1}$. The end points of the $m-1$ chords of $T_{m-1}$ partition the external circle of the chord diagram in $2(m-1)$ arcs, see Figure 26. Each of the rest of the chords of the chord diagram will begin and end in one of these arcs. If one of these chords begins and ends in the same arc, then it, together with $T_{m-1}$, is a set of $m$ nonintersecting chords. If not, there are $\left(\begin{array}{c}2(m-1) \\ 2\end{array}\right)$ many possibilities 
for the beginnings and ends of the extra arcs. Using the pigeonhole principle, if

$$
c m^{3}-(m-1)>\left(\begin{array}{c}
2(m-1) \\
2
\end{array}\right)(m-1)+1,
$$

we can always assume that there are $m$ many of the rest of the chords that all begin in one arc, and end in another. Let $\alpha, \beta$ be two arcs in which (at least) $m$ of the rest of the chords begin and end. Let us declare one of these chords to be "special" if it has one end in $\alpha$ and the other in $\beta$; otherwise declare it "nonspecial".

Let us look at the chords that begin in the arc $\alpha$. According to step 4 below, we can always move a "special" over a "nonspecial" one, and according to step 5 below, we can always move a "special" over another "special". After doing so, we may assume that the "special" $m$ chords do not intersect, and therefore, form an $m$-tower. q.e.d.
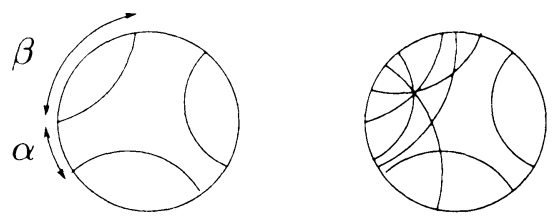

Figure 26. Shown on the left is a 3 tower, and two $\operatorname{arcs} \alpha, \beta$ of the external circle of the chord diagram. Shown on the right are some of the extra chords.

- Step 4 With the notation of step 3, we can always move a "special" chord over a "nonspecial" one.

Proof. The proof uses the 4-term relation. Fix a "special" chord and move a "nonspecial" in four ways around the end of the "special" one. Of the resulting four terms, two of them no longer have the "nonspecial" chord, and the two others move the "special" one over the nonspecial one. See Figure 27. q.e.d.

- Step 5 With the notation of step 3, we can always move a "special" chord over a "special" one. 


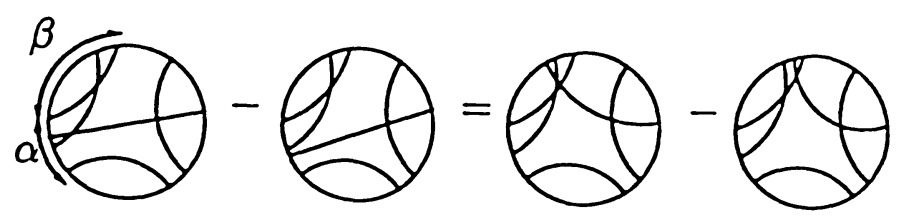

Figure 27. The 4-term relation with a fixed "special" chord and a moving "nonspecial" chord. For the convenience, the arcs $\alpha, \beta$ are shown, too. The first two terms show a "nonspecial" chord after and before passing a special chord (in the $\alpha$ arc). The two last terms have no "nonspecial" chord.

Proof. The same as in step 4, see Figure 28. q.e.d.

\section{- Step 6 Induction.}

We can now finish the proof of Proposition 3.13 as follows: obviously, a chord diagram contains (and therefore, is represented by) a 1-tower. Using step 3 and induction, Proposition 3.13 follows. q.e.d.

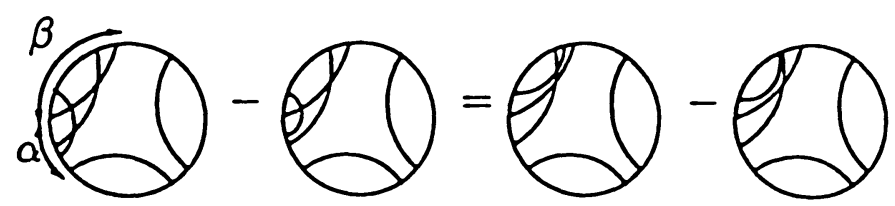

Figure 28. The 4-term relation with a fixed "special" chord and a moving "special" chord. For the convenience, the arcs $\alpha, \beta$ are shown, too. The first two terms show a "special" chord after and before passing a fixed "special" chord (in the $\alpha$ arc). The two last terms have chords that begin and end in the $\beta$ arc, and therefore, by the discussion of step 3 , we can find a 4 -tower.

We also have the following proposition, similar to, but different from Proposition 3.14:

Proposition 3.16. There is an increasing function $h: \mathbb{N} \rightarrow \mathbb{N}$ with the following property: for every $A S$-admissible link $L$ in $S^{3}$ (with 
framing $f$ ) which contains a sublink $L_{t r}(h(m)) \cup L^{\prime}$ with the following properties:

- $L_{t r}(h(m))$ is a trivial link of $h(m)$ components which bounds a disjoint union of discs $\cup_{i} D_{i}$.

- Each disc $D_{i}$ intersects the link $L^{\prime}$ in either two or four points. Moreover, the intersections of the disc $D_{i}$ with $L^{\prime}$ come in pairs with opposite orientation for each component of $L^{\prime}$. Furthermore, in case a disc $D_{i}$ intersects $L^{\prime}$ in four points, we assume that these four points do not lie in the same component of $L^{\prime}$. See Figure 29.

Then we have that $\left[S^{3}, L, f\right] \in \mathcal{F}_{m}^{b l} \mathcal{M}$.
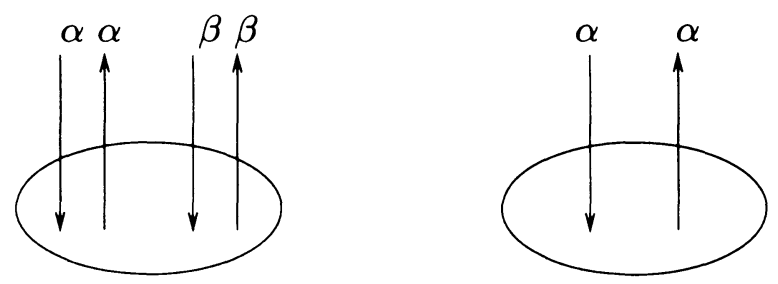

Figure 29. Shown here are the two allowable types of intersections of a disc $D_{i}$ with the components of $L^{\prime}$. Here $\alpha, \beta$ denote components of $L^{\prime}$ and we assume that $\alpha \neq \beta$.

Remark 3.17. Before we give the proof of the above proposition let us point out that the assumptions are weaker than those of Proposition 3.14. As a result, the conclusion is weaker than that of Proposition 3.14, in the sense that $\left[S^{3}, L, f\right]$ lies in $\mathcal{F}_{m}^{b l} \mathcal{M}$ and not necessarily in $\mathcal{F}_{m}^{b} \mathcal{M}$. Note also that the proof of Proposition 3.16 shows that the function $h$ is constructible, e.g. we can take $h(m)=c m^{13}$ for some constant $c$.

Proof of Proposition 3.16. Let $L, L^{\prime}$ be as in the statement of Proposition 3.16. We begin by introducing the associated chord diagram $C D_{L^{\prime}}$ of $L^{\prime}$ relative to the union of discs $D_{i}$. The chord diagram $C D_{L^{\prime}}$ consists of external circles (one per component of $L^{\prime}$ ) and chords (as many as the number of discs $D_{i}$ ). There are two types of chords: ones that intersect the external circles in two points, (called of type $I$ ) and the ones (called of type $I I$ ) that intersect the external circles in four points (however two points are in one circle and two are in another). For an example see Figure 30. Note that these chord diagrams are similar but 
different from the chord diagrams on links. At any rate, they include as a special case the chord diagrams considered in Proposition 3.14.

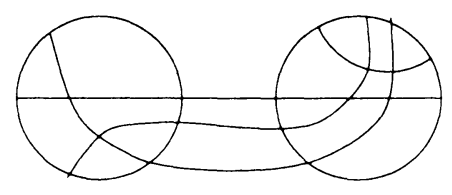

Figure 30. An example of a chord diagram considered in Proposition 3.16.

Two chords intersect if there is an external circle that they both touch, such that the four intersection points of that circle with the two chords is in the order 1212. See Figure 31. In analogy to Definition 3.15 we call a chord diagram $m$ - boundary if it contains $m$ nonintersecting chords. The motivation for considering nonintersecting chords, is the fact that they can be tubed, and therefore produce boundary links in the complement of $L^{\prime}$.
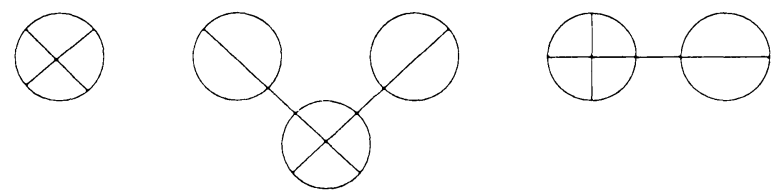

FIGURE 31. Examples of intersecting chords of various types: $(I, I),(I I, I I)$ and $(I, I I)$.

This shows the first step in the proof of Proposition 3.16:

- Step 1 If $C D_{L^{\prime}}$ is $m$-boundary, then $\left[S^{3}, L, f\right] \in \mathcal{F}_{m}^{b} \mathcal{M}$, and therefore (using Remark 1.13) $\left[S^{3}, L, f\right] \in \mathcal{F}_{m}^{b l} \mathcal{M}$.

The rest of the proof will be devoted to the proof that we can assume the hypothesis as in step 1. It uses, like Proposition 3.14, the 4-term relation in a crucial way. We sketch the proof here:

- Step 2 We can always assume that the framing in each of the components of $L_{t r}$ is +1 or -1 as we please.

Indeed, see step 2 of Proposition 3.14. We can now define (in direct analogy with Proposition 3.14) the notion of a chord diagram containing an $m$-tower. Let us concentrate on the chord diagram $C D_{L^{\prime}}$. With the above terminology we have the following: 
- Step 3 If the chord diagram $C D_{L^{\prime}}$ has an external circle which touches $g_{0}(m)>>m$ (for some function $g_{0}$ ), then it contains an $m$-tower, and therefore (by tubing) we conclude that $\left[S^{3}, L, f\right] \in$ $\mathcal{F}_{m}^{b l} \mathcal{M}$.

Before we give the proof, let us point out that the function $g_{0}$ above, (and the functions $g_{1}, g_{2}, h$ to be introduced later), can be constructed explicitly.

Proof. The proof uses the first, second and third version of the 4term relation on $\mathcal{M}$. (See Figures 19 and 23). Consider such an external circle, say $C_{1}$. Ignoring the subleading terms, apply the first, second and third versions of the 4-term relation (just as in step 3 of Proposition 3.14) in order to get $g_{1}(m)$ many chords, where $g_{0}(m)>c g_{1}(m)^{3}$, as in Step 3 of Proposition 3.14, which are nonintersecting as far as their ends in the external circle $C_{1}$ are concerned. Concentrate on the $g_{1}(m)$ many chords that touch the external circle $C_{1}$. Call these chords preferred. Consider all other external circles that these chords touch. Now we consider two cases:

Case 1 There are at least $m$ such other external circles.

Then we can create, using at least $m$ (of the $g_{1}(m)$ many preferred chords) an $m$-tower. See Figure 32 .

Case 2 Assume there are at most $m$ such external circles.

Then, if $g_{1}(m)>m g_{2}(m)$, there is at least one circle $C_{2}$ containing $g_{2}(m)$ many preferred chords (that lie on the circle $C_{1}$ and $C_{2}$ ). Ignoring the subleading terms once again, by applying the 4 -term relation, we can reach a linear combination of chord diagrams with a $3 m$-tower provided $g_{2}(m)>c(3 m)^{3}$.

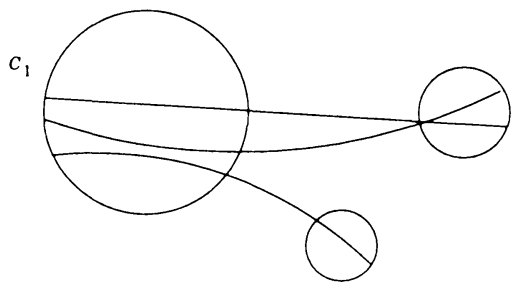

Figure 32. An example of an external circle $C_{1}$ and 3 chords that touch it and form a 3 -tower as far as this circle is concerned. 
This is all good, except that we used the 4-term relation twice, and we ignored the subleading terms in the 4-term relation twice. We need to deal with the subleading terms, too. Let's consider the subleading terms in the second case. The subleading terms of the 4-term relation contain an extra 1-pair blink in the complement of the rest of the link. However, the Seifert surface of this 1-pair blink may intersect the discs (i.e., whose boundaries are the chords of the chord diagram) of at most two chords. We call such chords marked. See Figure 21. Therefore each time we apply the 4-term relation, the subleading terms have an extra 1-pair blink and we mark at most two chords. Note that without loss of generality, the 4-term relation is applied to disjoint balls (embedded in $S^{3}$ ), and thus the 1-pair blinks bound surfaces disjoint from each other, and from the rest of the components of the link. Therefore, if in case 2 we apply the 4 -term relation more than $m$ times, the resulting subleading terms lie already in $\mathcal{F}_{m}^{b l} \mathcal{M}$. If on the other hand, we apply the 4-term relation at most $m$ times in order to create a $3 m$ tower, this means that we mark at most $2 m$ chords (of the preferred ones) and therefore have a subtower of $3 m-2 m=m$ chords. Similarly, we can deal with the subleading terms of the 4-term relation in the beginning of the proof. This concludes the proof of Step 3. q.e.d.

The above argument shows that ignoring the subleading terms in the 4-term relation does not affect the validity of our arguments. In the rest of the proof of Proposition 3.16 we will ignore such subleading terms. Due to step 3, let us assume that every external circle of the chord diagram $C D_{L^{\prime}}$ touches at most $g_{0}(m)$ many chords. If $C D_{L^{\prime}}$ has $h(m)>>g_{0}(m)$ chords, since every chord touches at most two circles, it implies that the number $n$ of external circles satisfies $n>>m$.

- Step 4 In this case, we have $\left[S^{3}, L, f\right] \in \mathcal{F}_{m}^{b l} \mathcal{M}$.

Proof. Fix a circle, and choose a chord $c_{1}$ that lies on the chosen circle. The chord touches at most two circles, and these circles have at most $2 g_{0}(m)$ many other chords that touch them. Mark all the circles that these chords touch and tube the chord $c_{1}$; see Figure 33. Now consider the rest of the circles (remembering that the number of circles is much greater than $m$ ), and proceed as above. q.e.d.

This concludes the proof of Proposition 3.16. Note that all the functions mentioned taking values in $\mathbb{N}$ are constructible. In fact, we leave it as an exercise to the reader to show that we can take $g_{2}(m)=$ 
$c_{2} m^{3}, g_{1}(m)=c_{1} m^{4}, g_{0}(m)=c_{0} m^{12}$ and $h(m)=c m^{13}$, for some constants $c_{0}, c_{1}, c_{2}, c$. q.e.d.

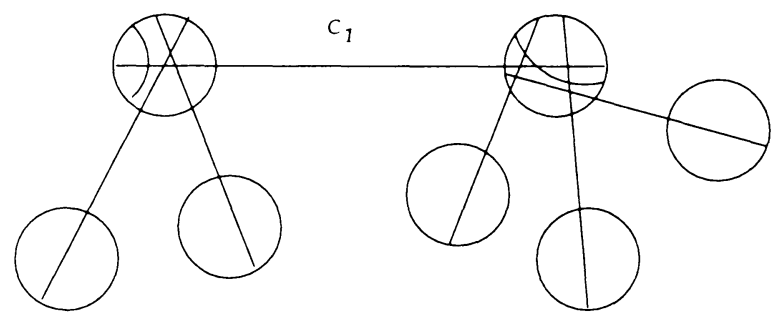

Figure 33. An example of a chord $c_{1}$ to be tubed and of the external circles that it marks.

Combining Propositions 3.13, 3.14 and 3.16 enables us to give a proof of theorem 3 as follows:

Proof of Theorem 3. Let $h$ be the function as in Proposition 3.16. We want to show that for every non-negative integer $m$, we have that $\mathcal{F}_{4 h(m)}^{a s} \mathcal{M} \subseteq \mathcal{F}_{m}^{b l} \mathcal{M}$. We know that $\mathcal{F}_{4 h(m)}^{a s} \mathcal{M}$ is spanned by all elements of the form $[M, L, f]$ where $(L, f)$ is an $A S$-admissible $4 h(m)$ - component link in an integral homology 3 -sphere $M$. For the convenience of the reader, we give the proof in three steps:

- Step 1 We may assume that $M=S^{3}$.

Indeed, it follows from the facts that: (i) every integral homology 3 -sphere can be obtained by surgery on an $A S$-admissible link $L^{\prime \prime}$ in $S^{3}$, (ii) the fundamental equation (13) and (iii) upward induction on the number of components of $L^{\prime \prime}$. See also [5, step 1, Theorem 1].

From now on, we assume that $(L, f)$ is an $A S$-admissible in $S^{3}$.

- Step 2 We may assume that $L$ contains a trivial sublink $L_{t r}(h(m))$ of $h(m)$ components.

Indeed, this is nothing but Proposition 3.13.

- Step 3 We may assume that the trivial sublink $L_{t r}(h(m))$ bounds a disjoint union of discs satisfying the properties of Proposition 3.16 . 
Indeed, since $L_{t r}(h(m))$ is a trivial link, it bounds a disjoint union of discs $U_{i} D_{i}$. Let $L^{\prime}$ be the sublink of $L$ that the union of the discs $D_{i}$ intersect. Since $L$ is an $A S$-admissible link, the intersections of the components of $L^{\prime}$ with each disc $D_{i}$ come in pairs with opposite orientations. Furthermore, using the equation shown in graphical notation in Figure 7 (for each of the discs $D_{i}$ ), we may assume that each disc intersects $L^{\prime}$ in two or four points. Furthermore, in case a disc $D_{i}$ intersects the same component of $L^{\prime}$ in four points, then Figure 34 shows that we can replace such intersections by a linear combination of discs that intersect that component in two points only. This finishes the proof of step 3, and together with Proposition 3.16 implies the proof of Theorem 3. q.e.d.

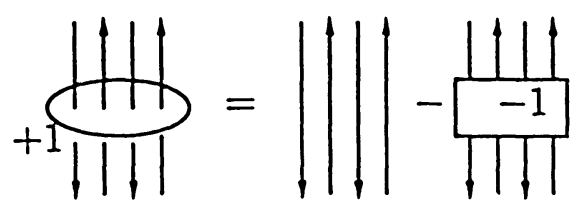

Figure 34. An equality in $\mathcal{M}$. The -1 in the box indicates a full twist. Notice that all arcs lie in the same link component, and that the two links shown on the right-hand side of the figure are homotopic, and therefore, by doing a number of crossing changes we can rewrite the right-hand side as a finite sum of terms each of which contains at least one disc that intersects the link component in two points.

\subsection{Proof of Theorem 4}

In this section we prove Theorem 4.

Proof of Theorem 4. We will first show that for every $m$ we have

$$
\mathcal{F}_{3 m}^{a s} \mathcal{M} \subseteq \mathcal{F}_{2 m}^{b l} \mathcal{M}+\mathcal{F}_{3 m+1}^{a s} \mathcal{M}
$$

This is equivalent to showing that $\mathcal{F}_{2 m}^{b l} \mathcal{M}$ spans the graded space $\mathcal{G}_{3 m}^{a s} \mathcal{M}$. However, we know a set of generators for this graded space, namely $[L(\Gamma)]$, where $\Gamma$ is a trivalent vertex-oriented graph with $3 m$ edges, and 
$L(\Gamma)$ is the associated $3 m$-component algebraically split link; see [22], and [5]. We will use a slightly different set of generators of this graded space namely $\left[L\left(\Gamma_{w}\right)\right]$, where

$$
\left[L\left(\Gamma_{w}\right)\right]=\sum_{v}[L(\Gamma(v))]
$$

where the sum is over all subsets of vertices of $\Gamma$, and $\Gamma(v)$ is the result of breaking the vertices of $\Gamma$ in $v$ according to Figure 35. Note that such vertices were called white in [9].

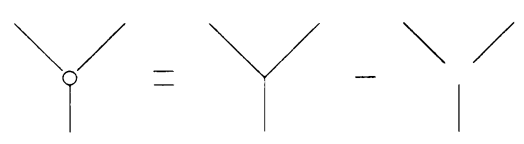

Figure 35. The definition of a white vertex. Note that each of the graphs represents a unit-framed algebraically split link in $S^{3}$.

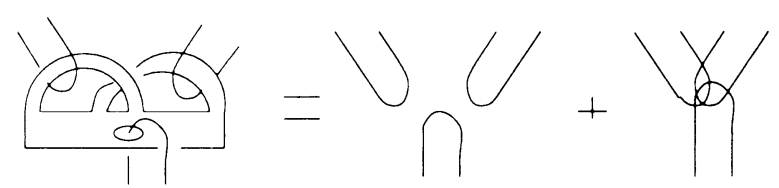

FIgURE 36. This figure represents a special case of equation (13) in graphical notation. The present identity holds in $\mathcal{G}_{*}^{a s} \mathcal{M}$. On the left shown is a 1-pair blink, which (after surgery) corresponds in $\mathcal{M}$ to the difference of two terms. The first term is shown on the first part on right, and the second term is (surgically equivalent to) the result of blowing down the 1-pair blink. Notice that each of the two components of the blink is an unknot and can be blown down in any order.

Using the identity in Figure 36 we see that summing over each white vertex (in the sum of $\left[L\left(\Gamma_{w}\right)\right]$ ) is equivalent to summing over a 1-pair blink. Since the graphs $\Gamma$ are trivalent with $3 m$ edges, (and therefore of $2 m$ vertices), we have that $\left[L\left(\Gamma_{w}\right)\right] \in \mathcal{F}_{2 m}^{b l} \mathcal{M}$. This finishes the proof of equation 23. Now interpolating equation (23) implies that: $\mathcal{F}_{3 m}^{a s} \mathcal{M} \subseteq$ $\widehat{\mathcal{F}_{2 m}^{b l} \mathcal{M}}$, which finishes the first part of the theorem.

The second part follows immediately from the first, using Theorems 2 and $3 . \quad$ q.e.d. 


\subsection{Proof of Theorem 2}

This section is devoted to the proof of Theorem 2 .

The proof presented here is similar to the proof of Theorem 1 of [6]. It uses primary and secondary inductions as well as the identities (for not-necessarily algebraically split links) of Section 3.1. For the convenience of the reader, we separate the proof into 5 steps. We begin with some definitions that will be useful. A triple of links $T=\left(L, L_{b}, L_{b l}\right)$ in an integral homology 3 -sphere $M$ consists of an algebraically split link $L$, a boundary link $L_{b}$ and a blink $L_{b l}$ such that each component of $L_{b}$ and pair of $L_{b l}$ bounds a connected oriented Seifert surface in $M$, and these surfaces are disjoint from each other and from $L$. Such a (disconnected) surface is called an admissible Seifert surface for $T$. If $k=|L|, n=\left|L_{b}\right|, m=\left|L_{b l}\right|$, then we call $T$ a $(k, n, m)-c l i n k$. The genus $g(T)$ is the minimal total genus of an admissible Seifert surface of $T$. An admissible framing for $T$ is one which is unit on $L \cup L_{b}$ and unit Seifert-framing on $L_{b l}$. We can then define $[M, T, f] \in \mathcal{M}$ in the usual way. We will prove that

$$
[M, T, f] \in \mathcal{F}_{3 n+3 m / 2}^{a s} \mathcal{M}
$$

Note that in [6, Theorem 1] we proved this fact in the special case $m=0$, and also that the present Theorem 2 is the case $n=0$ of equation (25). The argument for equation (25) is a generalization of that in [6], proceeding by primary downward induction on $k(T)=k$ and secondary upward induction on $g(T)$.

- Step 1 We may assume that $M=S^{3}$.

The proof follows from the following 3 facts:

- Every integral homology 3-sphere $M$ can be converted, by surgery on an $A S$-admissible link $L^{\prime}$, into $S^{3}$.

- We may assume that $L^{\prime}$ above can be chosen so that the Seifert surfaces bounded by $L_{b} \cup L_{b l}$ are disjoint from $L^{\prime}$. This follows by general position, since the Seifert surfaces are contained in a regular neighborhood of some embedded graph in $M$, and we can perturb $L^{\prime}$ away from this graph.

- Equation (13) 
and upward induction on the number of components of $L^{\prime}$, see also step 1 in Theorem 1 of [6].

Suppose now that $\left(L, L_{b}, L_{b l}\right)$ is a $(k, n, m)$-clink in $S^{3}$. If $k \geq 3 n+$ $3 m / 2$ we are done by definition. If $g(T)=0$ (i.e., $L_{b l}$ is an unblink, see Figure 1) we are also done, since in this case we have that $\left[S^{3}, T, f\right]=0$. Indeed, if $L_{b l}$ is a unit-Seifert framed unblink in an integral homology 3sphere $M$, then $\left[M, L_{b l}, f\right]=M-M_{L_{b l}, f}$, and by applying Kirby moves to a band of a genus 0 surface that $L_{b l}$ bounds, we deduce that $M_{L_{b l}, f}$ is diffeomorphic to $M$, and thus $\left[M, L_{b l}, f\right]=0$. This begins the induction.

- Step 2 We may assume that every component of $L$ is unknotted.

Equation (13) implies that the change of $\left[S^{3}, T, f\right]$ before and after a crossing change in the same component of $L$ can be written as $\left[S^{3}, T^{\prime}, f \cup \pm 1\right]$ where $T^{\prime}=\left(L \cup C, L_{b}, L_{b l}\right)$, and $C$ is a circle that encloses the crossing to be changed. Since $k\left(T^{\prime}\right)>k(T)$, by using the primary inductive hypothesis we can change crossings of components of $L$, and thus assume that each component of $L$ is unknotted.

- Step 3 Suppose that $L_{b} \cup L_{b l}=\partial \Sigma$, where $\Sigma$ is an admissible Seifert surface for $T$. We may assume that $\Sigma$ is embedded in a standard, almost planar (except for the necessary band crossings) way. See Figure 38.

This follows using the same argument as in [6] by introducing extra components into $L$ in order to change band crossings.

Let $\left\{K_{i}\right\}$ (for $1 \leq i \leq k(T)$ ) denote the components of $L$. Since by step 2 they are unknotted, we may choose embedded disks $D_{i}$ so that $K_{i}=\partial D_{i}$. Furthermore, since $\Sigma$ is just a thickening of a wedge of circles, we may choose the $D_{i}$ so that their intersections with $\Sigma$ consist of a number of transverse penetrations of the interiors of the $D_{i}$ by the bands of $\Sigma$. See Figure 37. We will be interested in counting the number of "band penetrations".

- Step 4 We may assume that every band of $\Sigma$ penetrates at least one $D_{i}$.

Proof. This follows by an argument similar to that in [6]. If some band penetrates no $D_{i}$, then we may arrange, as in [6], that the circle 


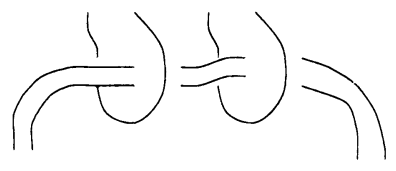

Figure 37. A band of a surface penetrating two pieces of discs.

in $\Sigma$ going through that band bounds a disk in $S^{3}$ disjoint from $L \cup \Sigma$. Therefore, depending on the band, we can either reduce the genus, as in [6], or remove one component of that pair. Thus we have turned one of the blink components into a bounding component and eliminated the band with no penetrations. If $T^{\prime}$ is the new clink, then it is easy to see that $\left[S^{3}, T^{\prime}, f \mid T^{\prime}\right]=\left[S^{3}, T, f\right]$. q.e.d.

- Step 5 We may assume that each disc $D_{i}$ has at most two band penetrations.

This follows precisely as in [6].

- Step 6 If any component $\Sigma_{j}$ of the Seifert surface of $L_{b}$ has genus one and a band of $\Sigma_{j}$ penetrates only one disk $D_{i}$, then we may assume that $D_{i}$ is penetrated by no other bands of $\Sigma$.

Again this will follow by the same argument as in [6].

We can now complete the proof of Theorem 2 by counting the band penetrations. Suppose that $T$ is a $(k, n, m)$ - clink satisfying all the assertions of the previous steps. Since $\left[S^{3}, T, f\right] \in \mathcal{F}_{k+n}^{a s} \mathcal{M}$, it suffices to show that $k \geq 2 n+3 m / 2$. Let $b$ be the number of penetrations of $\cup D_{i}$ by bands of $\Sigma$. Set $n=n_{0}+n_{1}$, where $n_{0}$ is the number of Seifert surface components for $L_{b}$ of genus one. Set $2 n_{0}=n_{0}^{\prime}+n_{0}^{\prime \prime}$ where $n_{0}^{\prime}$ is the number of bands of these genus-one Seifert surfaces with only one disk penetration.

Now it follows from Steps 4 and 6 that

$$
b \geq 3 m+4 n_{1}+n_{0}^{\prime}+2 n_{0}^{\prime \prime} .
$$

If we write $k=n_{0}^{\prime}+k^{\prime}$, then it follows from Step 5 that $b \leq n_{0}^{\prime}+2 k^{\prime}$. Combining these two inequalities gives

$$
n_{0}^{\prime}+2 k^{\prime} \geq 3 m+4 n_{1}+n_{0}^{\prime}+2 n_{0}^{\prime \prime}
$$


But then we have

$$
2 k=2 n_{0}^{\prime}+2 k^{\prime} \geq 3 m+4 n_{1}+2 n_{0}^{\prime}+2 n_{0}^{\prime \prime}=3 m+4 n,
$$

which was to be proved. The proof of Theorem 2 is complete. q.e.d.

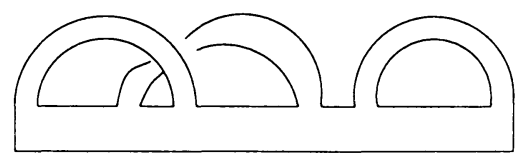

FIgURE 38. An example of a 1-pair blink that bounds a genus 1 surface. Note that the surface has 3 bands.

Remark 3.18. The bound obtained in Theorem 2 is sharp. Indeed, if $T$ is as in Figure 39, then $\left[S^{3}, T, f\right] \in \mathcal{F}_{3}^{a s} \mathcal{M}$, by Theorem 2, but we claim that $\left[S^{3}, T, f\right] \notin \mathcal{F}_{4}^{\text {as }} \mathcal{M}$. Indeed, using Figures 35 and 36 we see that Figure 39 represents the element $[\Theta] \in \mathcal{F}_{3}^{a s} \mathcal{M}$, where $[\Theta] \in \mathcal{M}$ is the element represented by the trivalent graph $\Theta$, with white vertices. But this element of $\mathcal{F}_{3}^{a s} \mathcal{M}$ is nontrivial in $\mathcal{G}_{3}^{a s} \mathcal{M}$, see [9, Proposition 2.13], or Proposition [5, Theorem 6]. This implies in particular that the analogue of the key Lemma 2.1 of [6] for blinks is false, and that the last step 6 of Theorem 1 of [6] would be false for blinks.

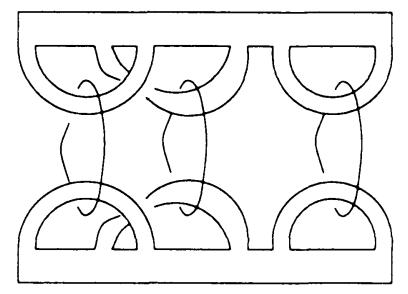

FIgURE 39. A special case of a 2-pair blink $L_{b l}$ union a 3component algebraically split link $L$. The result $\left[S^{3}, T, f\right]$ lies in $\mathcal{F}_{3}^{a s} \mathcal{M}$ and is non-trivial in $\mathcal{G}_{3}^{a s} \mathcal{M}$.

\section{Surgery equivalence and the Seifert matrix}

In this section we prove Theorem 5. This theorem suggests that finite type invariants in the sense of blinks, i.e., corresponding to the I-adic filtration of the Torelli group, according to Theorem 2, should be 
expressible in terms of Seifert matrix invariants of the associated blinks. Thus Alexander polynomial type invariants rather than Jones polynomial type invariants should suffice. This is an intriguing consequence which we hope to exploit in future work.

It is clear that surgery equivalent $n$-pair blinks (with framings which correspond) represent the same element of $\mathcal{G}_{n}^{b l} \mathcal{M}$.

Recall the notion of Seifert matrix of an oriented boundary link. If $L=\left(K_{1}, \cdots, K_{n}\right)$ and $K_{i}=\partial V_{i}$ where $\left\{V_{i}\right\}$ are disjoint oriented surfaces in an integral homology 3 -sphere $M$, then the Seifert pairing is the collection of bilinear pairings $\sigma_{i j}: H_{1}\left(V_{i}\right) \times H_{1}\left(V_{j}\right) \rightarrow \mathbb{Z}$ defined by $\sigma_{i j}(\alpha, \beta)=\operatorname{lk}\left(\alpha_{+}, \beta\right)$, the linking number, where $\alpha_{+} \in H_{1}\left(M-\cup_{i} V_{i}\right)$ represents the translate of $\alpha$ off $V_{i}$ in the positive normal direction. We can represent the Seifert pairing by a square integral matrix $A$ (the Seifert matrix) divided into blocks, each of which represents one of the $\sigma_{i j}$. There is an explicit algebraic description of the relation between two Seifert matrices of the same link corresponding to different choices of $\left\{V_{i}\right\}$ and different bases of the homology. This description uses the notion of S-equivalence and the action of a certain group of automorphisms of the free group (see [16] for the details).

The definition of a Seifert pairing of a blink is exactly the same using Seifert surfaces of the blink as defined in Definition 1.8. The Seifert pairing is again represented by a square matrix $A$ separated into blocks representing the $\sigma_{i j}$. The relation between two Seifert matrices of the same blink will be generally similar to that for boundary links, but more complicated, since it is permissible to change the orientation any of the Seifert surfaces. We do not want to explore this question now and our formulation of Theorem 5 allows us to avoid it.

\subsection{Proof of Theorem 5}

We first show that surgery equivalent blinks admit equal Seifert matrices. Suppose $(M, L)$ and $\left(M^{\prime}, L^{\prime}\right)$ are surgery equivalent. We may assume that they are related by a single blink surgery, i.e., if $\Sigma$ is a Seifert surface for $L$ in $M$, then there is a 1- pair blink $\left(l, l^{\prime}\right)$ in $M$ so that $l-l^{\prime}=\partial \sigma$ where $\sigma \subseteq M-\Sigma$ is a Seifert surface for $\left(l, l^{\prime}\right)$, and that $\left(M^{\prime}, L^{\prime}\right)=\left(M_{\left(l, l^{\prime}\right)}, L\right)$ using some unit Seifert-framing of $\left(l, l^{\prime}\right)$. Now we may also regard $\Sigma$ as a Seifert surface for $L^{\prime}$ in $M^{\prime}$, and so we need to show that if $\alpha, \beta \in H_{1}(\Sigma)$, then $\mathrm{lk}_{M}\left(\alpha_{+}, \beta\right)=\mathrm{lk}_{M^{\prime}}\left(\alpha_{+}, \beta\right)$ where $\mathrm{lk}_{M}$ denotes the linking number in $M$. In general, given two disjoint simple closed curves $\xi, \eta$ in $M-\Sigma$, we show that their linking number in $M$ 
is the same as in $M^{\prime}$. Suppose $\eta$ bounds an orientable surface $\Lambda$ in $M$. By general position $\sigma \cap \Lambda$ is a collection of proper curves in $\sigma$ and so, homologically, $\eta=\operatorname{lk}(\xi, \eta) m+r\left(m_{l}+m_{l^{\prime}}\right)$ in $M-\xi-l-l^{\prime}$, where $m$ is a meridian of $\xi, m_{l}$ and $m_{l^{\prime}}$ are meridians of $l$ and $l^{\prime}$, and $r$ is some integer. Thus it suffices to observe that $m_{l}+m_{l^{\prime}}$ is homologically trivial in $M^{\prime}-\xi$. But, by the definition of a unit Seifert framing $m_{l}^{\prime}=m_{l}+\epsilon l$ and $m_{l^{\prime}}^{\prime}=m_{l^{\prime}}-\epsilon l^{\prime}$, for some $\epsilon= \pm 1$, and so

$$
m_{l}+m_{l^{\prime}}=m_{l}^{\prime}+m_{l^{\prime}}^{\prime}+\epsilon\left(l^{\prime}-l\right)=m_{l}^{\prime}+m_{l^{\prime}}^{\prime}+\epsilon \partial \Sigma=0 \in H_{1}\left(M^{\prime}-\xi\right) .
$$

\subsection{Conclusion of proof}

Now suppose that two blinks $(M, L)$ and $\left(M^{\prime}, L^{\prime}\right)$ admit the same Seifert matrix. We may first of all assume that $M=M^{\prime}=S^{3}$ since, by Proposition 1.16, we can convert any 3 -manifold into $S^{3}$ by surgery on a boundary link. which we can assume is far away from any other given link. By the observation in Remark 1.13 this is the same as surgery on some blink. Let $\Sigma, \Sigma^{\prime}$ be Seifert surfaces for $L, L^{\prime}$ which give identical Seifert matrix $A$. Since $A-A^{T}$ is the intersection matrix of $\Sigma$ and $\Sigma^{\prime}$, we conclude that $\Sigma$ and $\Sigma^{\prime}$ are diffeomorphic.

Now it is an easy consequence of Smale theory that the regular homotopy type of an embedding of a bounded surface in $S^{3}$ is determined by the twisting numbers of the bands mod 2. Since these twisting numbers are determined by the Seifert matrix, it follows that $\Sigma$ and $\Sigma^{\prime}$ are regularly homotopic. A regular homotopy of $\Sigma$ consists of a sequence of isotopies and crossings of bands (see Figure 40), so we only have to show that the band crossings can be achieved by blink surgeries.

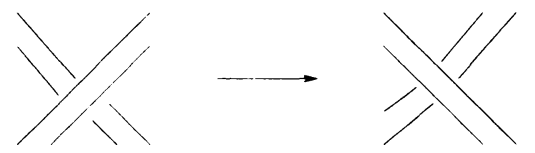

Figure 40. An illustration of a crossing of bands.

Let $b_{1}, b_{2}$ be any two bands of $\Sigma$, possibly the same band. Each time when we encounter a crossing of $b_{1}$ with $b_{2}$, there is a corresponding change in the Seifert matrix. Since the net change in the Seifert matrix must be zero, we conclude that there is an equal number of crossings of $b_{1}$ and $b_{2}$ in each of the two directions. Now suppose that our regular homotopy actually breaks up into a sequence of isotopies and double band crossings, where we define a double band crossing as 
two simultaneous crossings of a single pair of bands in opposite directions (see Figure 41). We illustrate in Figure 42 that a double band crossing can be achieved by a blink surgery.

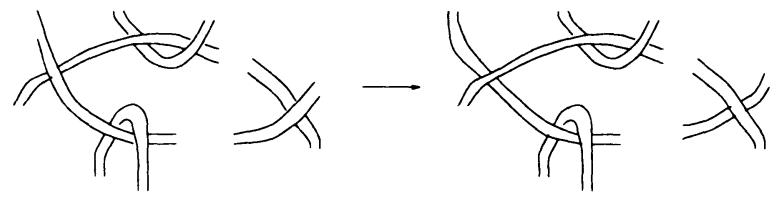

Figure 41. A double crossing change of bands in opposite directions.

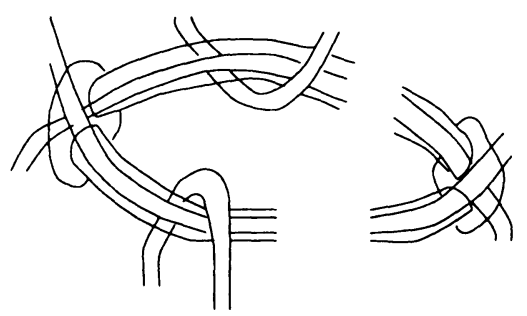

FIgURE 42. A 1-pair blink that achieves the double crossing change of the bands of Figure 41.

We need to do two oppositely framed surgeries on circles around the two bands at the crossing points. The surface bounded by these two circles is obtained by taking the small twice punctured disks bounded by these circles and connecting the punctures of one with the punctures of the other by tubes along the two band segments connecting the crossing points.

So it suffices to show that we can find a regular homotopy from $\Sigma$ to $\Sigma^{\prime}$ consisting of isotopies and double band crossings. Let us consider $\Sigma$ and $\Sigma^{\prime}$ as disks $D$ and $D^{\prime}$ with bands attached. By a preliminary isotopy we can assume that $D=D^{\prime}$ and even a bit more, that $\Sigma$ and $\Sigma^{\prime}$ coincide in a neighborhood of $D=D^{\prime}$. Then we can choose a regular neighborhood $N$ of $D$, and assume that we have a regular homotopy which moves the bands of $\Sigma$ onto those of $\Sigma^{\prime}$ in the complement of $N$ and is stationary inside $N$. Let us modify this regular homotopy of $\Sigma$ by performing some additional band moves inside $N$. Every time a band crossing occurs (outside $N$ ) let us introduce a crossing of the same bands, but in the opposite direction, inside $N$ (see Figures 43 and 
44). Thus every band crossing in the original homotopy is replaced by a double band crossing, and so our new regular homotopy will be a sequence of double band crossings. This new homotopy now consists of two independent parts: the original homotopy outside $N$ and the new part inside $N$. We have complete freedom in how we perform each of the band crossings inside $N$ and so, since the number of crossings of each pair of bands in the two directions is equal, we can choose the corresponding crossings introduced inside $N$ to cancel each other. Thus the net effect will be to leave $\Sigma \cap N$ unchanged. In other words our modified regular homotopy will have the same result as the original regular homotopy, i.e., to move $\Sigma$ onto $\Sigma^{\prime}$. Since the modified regular homotopy is a sequence of double band crossings, this completes the proof. q.e.d.
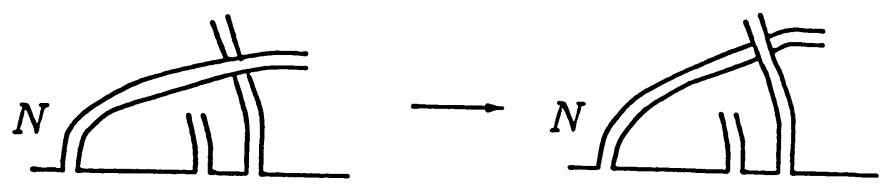

FIgURE 43. An illustration of an original homotopy.
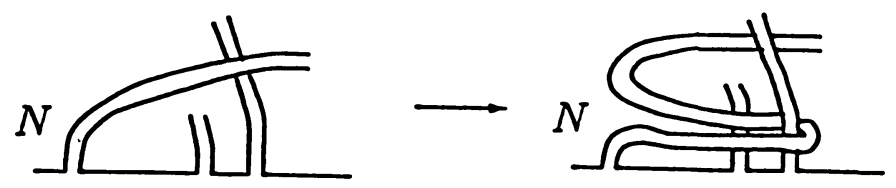

Figure 44. An illustration of a modified homotopy. 


\section{Appendix}

\subsection{Remarks on the group $\mathcal{L}_{g, 1}$}

If $L \subseteq H=H_{1}\left(\Sigma_{g}\right)$ is the Lagrangian used to define $\mathcal{L}_{g, 1}$, then we can also define a larger group $\overline{\mathcal{L}}_{g, 1}=\left\{h: h_{*} \mid L=\right.$ identity $\}$, where $h_{*}$ is the automorphism of $H$ induced by $h$. Clearly $\mathcal{L}_{g, 1} \subseteq \overline{\mathcal{L}}_{g, 1}$ and $\mathcal{T}_{g, 1} \subseteq \overline{\mathcal{L}}_{g, 1}$. So we have a lattice of subgroups of the mapping class group:

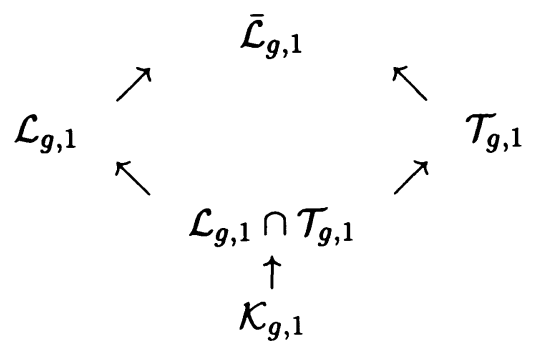

Note that the above diagram defines a map

$$
\mathcal{L}_{g, 1} / \mathcal{L}_{g, 1} \cap \mathcal{T}_{g, 1} \rightarrow \overline{\mathcal{L}}_{g, 1} / \mathcal{T}_{g, 1}
$$

We can now show the following:

Proposition 5.1. The above defined map

$$
\mathcal{L}_{g, 1} / \mathcal{L}_{g, 1} \cap \mathcal{T}_{g, 1} \rightarrow \overline{\mathcal{L}}_{g, 1} / \mathcal{T}_{g, 1}
$$

is an isomorphism.

Proof. By its definition it follows that it is one-to-one. In order to show that it is onto, recall first [10], [12], [20] the following classical short exact sequence:

$$
1 \rightarrow \mathcal{T}_{g, 1} \rightarrow \Gamma_{g, 1} \rightarrow S p(2 g, \mathbb{Z}) \rightarrow 1,
$$

where the map $\Gamma_{g, 1} \rightarrow S p(2 g, \mathbb{Z})$ is the map $h \rightarrow h_{*}$ that sends a surface diffeomorphism to its linear action on $H_{1}\left(\Sigma_{g}, \mathbb{Z}\right)$. We therefore have an isomorphism $\Gamma_{g, 1} / \mathcal{T}_{g, 1} \simeq S p(2 g, \mathbb{Z})$. We can therefore identify $\overline{\mathcal{L}}_{g, 1} / \mathcal{T}_{g, 1}$ with its image in $S p(2 g, \mathbb{Z})$, and as such, $\overline{\mathcal{L}}_{g, 1} / \mathcal{T}_{g, 1}$ consists of all isometries $\phi$ of $H_{1}\left(\Sigma_{g}\right)$ which are the identity on $L$. With these preliminaries in mind, in order to show that the map of the proposition is onto, it suffices to show that every isometry $\phi$ of $H_{1}\left(\Sigma_{g}\right)$ which is the identity on $L$ is induced by some product $\rho_{1} \cdots \rho_{k}$ of $L$-twists. If 
we write $H_{1}\left(\Sigma_{g}\right)=L \oplus L^{\prime}$ where $L^{\prime}$ is a Lagrangian dual to $L$, and we choose a basis $\left\{e_{i}\right\}$ of $L$ and dual basis $\left\{e_{i}^{\prime}\right\}$ for $L^{\prime}$, then $\phi$ has a matrix representative: $\left(\begin{array}{ll}I & C \\ 0 & I\end{array}\right)$, where $C$ is a symmetric matrix. If $\rho$ is a Dehn twist along a simple closed curve representing $\sum_{i} \lambda_{i} e_{i}$, then it has such a matrix representative, where the entries of $C$ are given by $c_{i j}= \pm \lambda_{i} \lambda_{j}$. We can certainly realize the elements $e_{i}$ and $e_{i} \pm e_{j}$ by simple closed curves, and it is then an easy exercise to see that any $C$ can be realized by a composition of Dehn twists along such curves, using the fact that

$$
\left(\begin{array}{ll}
I & C \\
0 & I
\end{array}\right) \cdot\left(\begin{array}{cc}
I & C^{\prime} \\
0 & I
\end{array}\right)=\left(\begin{array}{cc}
I & C+C^{\prime} \\
0 & I
\end{array}\right)
$$

q.e.d.

Notice also that $\left[\overline{\mathcal{L}}_{g, 1}, \overline{\mathcal{L}}_{g, 1}\right] \subseteq \mathcal{T}_{g, 1}$ because $\overline{\mathcal{L}}_{g, 1} / \mathcal{T}_{g, 1}$ is abelian

The next natural problem to consider is the determination of $\mathcal{L}_{g, 1} \cap$ $\mathcal{T}_{g, 1} / \mathcal{K}_{g, 1}$. In order to do so, we will need an important homomorphism $\tau: \mathcal{T}_{g, 1} \rightarrow \Lambda^{3} H$ defined by $\mathrm{D}$. Johnson (see [13]). We review its definition here: If $h \in \mathcal{T}_{g, 1}$ then, by definition, $h_{*}$ is the identity on $H$. Thus, for any $\beta \in \pi=\pi_{1}\left(\Sigma_{g}\right)$, we can write:

$$
h_{*}(\beta) \beta^{-1} \equiv t(h) \cdot \beta \in \pi_{2} / \pi_{3} \simeq \Lambda^{2} H .
$$

This defines a homomorphism $t: \mathcal{T}_{g, 1} \rightarrow \operatorname{hom}\left(H, \Lambda^{2} H\right)$. We now have the identifications:

$$
\operatorname{hom}\left(H, \Lambda^{2} H\right) \simeq H^{*} \otimes \Lambda^{2} H \simeq H \otimes \Lambda^{2} H,
$$

where the latter isomorphism uses the symplectic structure on $H$. Thus we obtain a homomorphism $t^{\prime}: \mathcal{T}_{g, 1} \rightarrow H \otimes \Lambda^{2} H$. Johnson showed that $\operatorname{im}\left(t^{\prime}\right) \subseteq \Lambda^{3} H$, where the embedding $\Lambda^{3} H \subseteq H \otimes \Lambda^{2} H$ is defined by:

$$
x \wedge y \wedge z \mapsto x \otimes(y \wedge z)+y \otimes(z \wedge x)+z \otimes(x \wedge y) .
$$

This defines a homomorphism $\tau: \mathcal{T}_{g, 1} \rightarrow \Lambda^{3} H$. Johnson showed that $\tau$ is onto and its kernel is exactly $\mathcal{K}_{g, 1}$.

With these preliminaries in mind, an important step in understanding $\mathcal{L}_{g, 1}$ is the calculation of $\tau\left(\mathcal{L}_{g, 1} \cap \mathcal{T}_{g, 1}\right)$. For example, by Proposition 5.1, $\tau\left(\mathcal{L}_{g, 1} \cap \mathcal{T}_{g, 1}\right)=\Lambda^{3} H$ if and only if $\mathcal{L}_{g, 1}=\overline{\mathcal{L}}_{g, 1}$. But we now show that this is false.

Proposition 5.2. Suppose $h \in \mathcal{T}_{g, 1} \cap \mathcal{L}_{g, 1}$. Then

$$
\tau(h) \in \operatorname{ker}\left\{\Lambda^{3} H \rightarrow \Lambda^{3}(H / L)\right\} \text {. }
$$


Proof. Let $h \in \mathcal{T}_{g, 1} \cap \mathcal{L}_{g, 1}$. With the discussion of Johnson's homomorphism above, and using the the following commutative diagram:

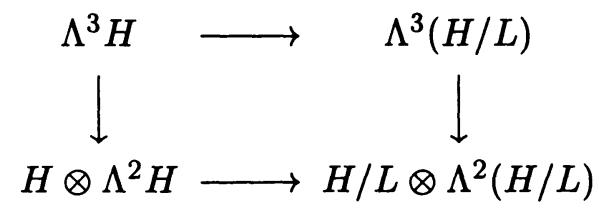

(where the vertical arrows are both injections, and $L$ is the Lagrangian used to define $\left.\mathcal{L}_{g, 1}\right)$ it follows that the proposition is equivalent to showing that $t^{\prime}(h) \in \operatorname{ker}\left\{H \otimes \Lambda^{2} H \rightarrow H / L \otimes \Lambda^{2}(H / L)\right\}$ or, equivalently, $t(h) \in \operatorname{ker}\left\{\operatorname{hom}\left(H, \Lambda^{2} H\right) \rightarrow \operatorname{hom}\left(L, \Lambda^{2}(H / L)\right\}\right.$. In order to show that we need the following lemma:

Lemma 5.3. Suppose that $\Sigma$ is a compact orientable surface with one boundary component, and $C$ a simple closed curve in the interior of $\Sigma$ representing, up to conjugacy, an element $\alpha \in \pi=\pi_{1}(\Sigma)$. Let $h$ denote the homeomorphism of $\Sigma$ defined by a Dehn twist along $C$. Then, for any $\beta \in \pi$, we can write

$$
h_{*}(\beta) \beta^{-1}=\gamma_{1}^{\epsilon_{1}} \cdots \gamma_{k}^{\epsilon_{k}}
$$

where each $\gamma_{i}$ is a conjugate of $\alpha$ and, if $[\xi]$ denotes the homology class of $\xi$ for any $\xi \in \pi$, then $\sum_{i} \epsilon_{i}= \pm[\alpha] \cdot[\beta]$, the intersection number, and the sign depends on the direction of the Dehn twist.

Proof. If $\lambda$ is any path in $\Sigma$ which intersects $C$ transversely, then we can write $h \circ \lambda$ as a product $\lambda_{1} \cdot C^{\epsilon_{1}} \cdots \lambda_{k} \cdot C^{\epsilon_{k}} \cdot \lambda_{k+1}$, for some factorization $\lambda=\lambda_{1} \cdots \lambda_{k+1}$ as a product of paths. The $C^{\epsilon_{i}}$ insert themselves whenever $\lambda$ crosses $C$, and $\epsilon_{i}$ is the sign of the intersection. q.e.d.

We can rewrite $h_{*}(\beta) \beta^{-1}$ as given in Lemma 5.3 in the form

$$
\left[\xi_{1}, \alpha^{\epsilon_{1}}\right] \alpha^{\epsilon_{1}} \cdots\left[\xi_{k}, \alpha^{\epsilon_{k}}\right] \alpha^{\epsilon_{k}} \equiv\left(\prod_{i}\left[\xi_{i}, \alpha^{\epsilon_{i}}\right]\right) \alpha^{e} \bmod \pi_{3}
$$

where $e= \pm[\alpha] \cdot[\beta]$. In particular hold in mind the case of $e=0$. In this case if we apply another homeomorphism defined by a Dehn twist along a curve representing $\gamma \in \pi$ so that $[\alpha] \cdot[\gamma]=0$, then $h_{*}(\beta) \beta^{-1}$ is mapped to a new element which is still in the form of equation (30) with $e=0$. Continuing in this way we obtain the following conclusion: suppose that 
$h$ is a product of Dehn twists on curves representing elements in some Lagrangian $L$. If $\beta \in \pi$ also represents an element of $L$, then

$$
h_{*}(\beta) \beta^{-1} \equiv \prod_{i}\left[\xi_{i}, \alpha_{i}\right] \bmod \pi_{3},
$$

where $\left[\alpha_{i}\right] \in L$. But this shows that:

$$
t(h) \cdot \beta=\sum_{i}\left[\xi_{i}\right] \wedge\left[\alpha_{i}\right]
$$

Since $\left[\alpha_{i}\right] \in L$, the right side clearly maps to 0 in $\Lambda^{2}(H / L)$. q.e.d.

Proposition 5.1 immediately implies the following corollary:

Corollary 5.4. As a subgroup of the mapping class group, $\overline{\mathcal{L}}_{g, 1}$ is generated by $\mathcal{T}_{g, 1}$ and $\mathcal{L}_{g, 1}$.

Remark 5.5. $t \mid \mathcal{T}_{g, 1} \cap \mathcal{L}_{g, 1}$ actually extends to a homomorphism $\tilde{t}$ : $\overline{\mathcal{L}}_{g, 1} \rightarrow \operatorname{hom}\left(L, \Lambda^{2} H\right)$ by the same definition as for $t$, using the defining property that, if $h \in \overline{\mathcal{L}}_{g, 1}$ then $h_{*} \mid L=$ identity. The above proof actually shows that $\tilde{t}\left(\mathcal{L}_{g, 1}\right) \subseteq \operatorname{hom}(L, K)$, where $K=\operatorname{ker}\left\{\Lambda^{2} H \rightarrow \Lambda^{2}(H / L)\right\}$.

Question 6. Is $\mathcal{L}_{g, 1}=\tilde{t}^{-1} \operatorname{hom}(L, K)$ ? Is $\tau\left(\mathcal{L}_{g, 1} \cap \mathcal{T}_{g, 1}\right)=\operatorname{ker}\left\{\Lambda^{3} H \rightarrow\right.$ $\left.\Lambda^{3}(H / L)\right\}$ ?

\subsection{The lower central series of $\overline{\mathcal{L}}_{g, 1}$}

In this section we study the image of the lower central series of $\overline{\mathcal{L}}_{g, 1}$ under Johnson's map into $\Lambda^{3} H$. In particular we prove:

Proposition 5.6. For all $g \geq 1$ we have: $\left(\overline{\mathcal{L}}_{g, 1}\right)_{5} \subseteq \mathcal{K}_{g, 1}$. In addition, for $g \geq 1$ we have: $\left(\overline{\mathcal{L}}_{g, 1}\right)_{4} \nsubseteq \mathcal{K}_{g, 1}$ if $g \geq 3$.

The proof of Proposition 5.6 will be based on the following:

Lemma 5.7. With the notation of Remark 5.5, we have the following: $t^{\prime}\left(\left[\overline{\mathcal{L}}_{g, 1}, \overline{\mathcal{L}}_{g, 1}\right]\right) \subseteq\left(L \otimes \Lambda^{2} H\right)+(H \otimes K)$.

Using Corollary 5.4, and the fact that $\left[\mathcal{T}_{g, 1}, \mathcal{T}_{g, 1}\right] \subseteq \mathcal{K}_{g, 1}$, the lemma will follow from the following two assertions:

1. $t^{\prime}\left(\left[\mathcal{L}_{g, 1}, \mathcal{L}_{g, 1}\right]\right) \subseteq H \otimes K$.

2. $t^{\prime}\left(\left[\mathcal{L}_{g, 1}, \mathcal{T}_{g, 1}\right]\right) \subseteq(H \otimes K)+\left(L \otimes \Lambda^{2} H\right)$. 
Proof of (1). $\quad\left[\mathcal{L}_{g, 1}, \mathcal{L}_{g, 1}\right]$ is generated by elements of the form $\left[h, D_{C}\right]$, where $h \in \mathcal{L}_{g, 1}, C$ is a simple closed curve in $M$, which represents an element of $L$, and $D_{C}$ denotes a Dehn twist along $C$. Now $\left[h, D_{C}\right]=D_{h(C)} \circ\left(D_{C}\right)^{-1}$. We can apply Lemma 5.3 to obtain;

$$
\left(D_{C}\right)_{*}(\beta) \equiv \beta \prod_{i}\left[\xi_{i}, \alpha^{\epsilon_{i}}\right] \alpha^{d} \quad \bmod \pi_{3}
$$

where $C$ represents $\alpha \in \pi$, up to conjugation, and $d= \pm[\alpha] \cdot[\beta]$, for any $\beta \in \pi$. Similarly we have:

$$
\left(D_{h(C)}\right)_{*}(\beta) \equiv \beta \prod_{i}\left[\theta_{i}, h_{*}(\alpha)^{\epsilon_{i}^{\prime}}\right] h_{*}(\alpha)^{e} \quad \bmod \pi_{3}
$$

where $e= \pm\left[h_{*} \alpha\right] \cdot[\beta]$, for any $\beta \in \pi$. Putting these together we get:

$\left(D_{h(C)}\right)_{*} \circ\left(D_{C}\right)_{*}^{-1}(\beta) \equiv \beta \prod_{i}\left[\theta_{i}, h_{*}(\alpha)^{\epsilon_{i}^{\prime}}\right] \prod_{i}\left[\xi_{i}, \alpha^{\epsilon_{i}}\right] \alpha^{d} h_{*}(\alpha)^{e} \quad \bmod \pi_{3}$, where $d= \pm[\alpha] \cdot[\beta]$ again, but now $e= \pm\left[h_{*} \alpha\right] \cdot\left(D_{C}\right)_{*}^{-1}[\beta]$.

Since $h \in \mathcal{L}_{g, 1}$ and $[\alpha] \in L$, we have $h_{*}(\alpha) \alpha^{-1} \in \pi_{2}$ and so $\alpha$ and $h_{*}(\alpha)$ commute $\bmod \pi_{3}$. Thus equation (34) can be rewritten:

$$
\begin{aligned}
& \left(D_{h(C)}\right)_{*} \circ\left(D_{C}\right)_{*}^{-1}(\beta) \\
& \quad \equiv \beta \prod_{i}\left[\theta_{i}, h_{*}(\alpha)^{\epsilon_{i}^{\prime}}\right] \prod_{i}\left[\xi_{i}, \alpha^{\epsilon_{i}}\right]\left(h_{*}(\alpha) \alpha^{-1}\right)^{e} \alpha^{d+e} \quad \bmod \pi_{3} .
\end{aligned}
$$

This simplifies considerably to:

$$
\left(D_{h(C)}\right)_{*} \circ\left(D_{C}\right)_{*}^{-1}(\beta) \equiv \beta \alpha^{d+e} \quad \bmod \pi_{2} .
$$

But $D_{h(C)} \circ\left(D_{C}\right)^{-1} \in \mathcal{T}_{g, 1}$, since $h(C)$ is homologous to $C$, and so $d+e=0$. (We can assume that $[\alpha] \neq 0$, otherwise we already have that $D_{C} \in \mathcal{K}_{g, 1}$.)

We can apply equation (31) to write:

$$
h_{*}(\alpha) \alpha^{-1} \equiv \prod_{i}\left[\eta_{i}, \alpha_{i}\right] \bmod \pi_{3}
$$

where $\left[\alpha_{i}\right] \in L$. Putting this all together into equation (35) we get:

$$
\left(D_{h(C)}\right)_{*} \circ\left(D_{C}\right)_{*}^{-1}(\beta) \equiv \beta \prod_{i}\left[\zeta_{i}, \alpha_{i}^{\prime}\right]
$$


where $\left[\alpha_{i}^{\prime}\right] \in L$. This translates into:

$$
\tilde{t}\left(\left(D_{h(C)}\right) \circ\left(D_{C}\right)^{-1}\right) \cdot[\beta]=\sum_{i}\left[\zeta_{i}\right] \wedge\left[\alpha_{i}^{\prime}\right],
$$

and this element lies in $K$. q.e.d.

Proof of (2). Suppose $\lambda \in \overline{\mathcal{L}}_{g, 1}$ and $h \in \mathcal{T}_{g, 1}$. Then $[\lambda, h]=$ $\left(\lambda h \lambda^{-1}\right) h^{-1}$ which can be written, as an element of the abelianization $\mathcal{T}_{g, 1} /\left(\mathcal{T}_{g, 1}\right)_{2}$, in the additive form $\lambda \cdot h-h$, where we use the canonical action of the mapping class group $\Gamma_{g, 1}$ on $\mathcal{T}_{g, 1} /\left(\mathcal{T}_{g, 1}\right)_{2}$ by conjugation. It was pointed out by Johnson (see e.g. [13]) that $\tau$, or $t^{\prime}: \mathcal{T}_{g, 1} \rightarrow H \otimes \Lambda^{2} H$, is equivariant with respect to the action of $\Gamma_{g, 1}$ (acting on the right side by the canonical action on $H$ ). Thus

$$
t^{\prime}([\lambda, h])=t^{\prime}(\lambda \cdot h-h)=(\lambda-1) \cdot t^{\prime}(h) .
$$

If $a \otimes\left(a_{1} \wedge a_{2}\right) \in H \otimes \Lambda^{2} H$, then

$$
\begin{aligned}
(\lambda-1) \cdot\left(a \otimes\left(a_{1} \wedge a_{2}\right)\right)= & (\lambda-1) a \otimes \lambda\left(a_{1} \wedge a_{2}\right) \\
& +a \otimes\left((\lambda-1) a_{1} \wedge \lambda a_{2}\right) \\
& +a \otimes\left(a_{1} \wedge(\lambda-1) a_{2}\right) .
\end{aligned}
$$

Now recall that, for any $\lambda \in \overline{\mathcal{L}}_{g, 1}$, the action on $H$ satisfies:

- $\lambda \mid L=$ identity,

- $(\lambda-1)(H) \subseteq L$,

and so $(\lambda-1)^{2}=0$. Thus, in equation (37), the terms on the right side are in either $L \otimes \Lambda^{2} H$ or $H \otimes K$.

This completes the proof of (2) and of Lemma 5.7. q.e.d.

Proof of Proposition 5.6. We will use an argument similar to that in the proof of (2) above to prove the following assertions in order:

1. $t^{\prime}\left(\left(\overline{\mathcal{L}}_{g, 1}\right)_{3}\right) \subseteq(L \otimes K)+\left(H \otimes \Lambda^{2} L\right)$,

2. $t^{\prime}\left(\left(\overline{\mathcal{L}}_{g, 1}\right)_{4}\right) \subseteq\left(L \otimes \Lambda^{2} L\right)$

3. $t^{\prime}\left(\left(\overline{\mathcal{L}}_{g, 1}\right)_{5}\right)=0$.

Recalling Corollary 5.4, the above two assertions prove Proposition 5.6.

To prove (1) we apply equation (37), where we can assume, by Lemma 5.7, either $a_{1} \wedge a_{2} \in K$ or $a \in L$, and we see that the terms on 
the right side of equation (37) lie in $(L \otimes K)+\left(H \otimes \Lambda^{2} L\right)$. Note that $(\lambda-1) K \subseteq \Lambda^{2} L$.

To prove (2) (or (3)) we use equation (37) in the same way, taking into account (1) (or (2)) to tell where $a \otimes\left(a_{1} \wedge a_{2}\right)$ must lie.

This completes the proof that $\left(\tilde{\mathcal{L}_{g, 1}}\right)_{5} \subseteq \mathcal{K}_{g, 1}$. It remains to show that, if $g \geq 3$ then $t^{\prime}\left(\left(\overline{\mathcal{L}}_{g, 1}\right)_{4}\right) \neq 0$.

Suppose $h \in \mathcal{T}_{g, 1}$ so that $\tau(h)=a_{1} \wedge a_{2} \wedge a_{3} \in \Lambda^{3} H$. If $\lambda \in \mathcal{L}_{g, 1}$ then we have $\tau([\lambda, h])=(\lambda-1) \cdot\left(a_{1} \wedge a_{2} \wedge a_{3}\right)$. But we can use the following analogue of equation (37):

$$
\begin{aligned}
(\lambda-1) \cdot\left(a_{1} \wedge a_{2} \wedge a_{3}\right)= & (\lambda-1) a_{1} \wedge \lambda a_{2} \wedge \lambda a_{3} \\
& +a_{1} \wedge(\lambda-1) a_{2} \wedge \lambda a_{3} \\
& +a_{1} \wedge a_{2} \wedge(\lambda-1) a_{3} .
\end{aligned}
$$

Noting that $(\lambda-1) a_{i} \in L$ and $(\lambda-1) \mid L=0$, we can use equation (38) in this way repeatedly to compute:

$$
\tau([\lambda,[\lambda,[\lambda, h]]])=6(\lambda-1) a_{1} \wedge(\lambda-1) a_{2} \wedge(\lambda-1) a_{3}
$$

Now suppose, following the conventions in the proof of Proposition 5.1, that $\left\{e_{i}\right\}$ is a basis of $L$ and $\left\{e_{i}^{\prime}\right\}$ is a dual basis of $L^{\prime}$. For any symmetric matrix $C$ there is some $\lambda \in \mathcal{L}_{g, 1}$ so that:

$$
\lambda\left(e_{i}\right)=e_{i}, \quad \lambda\left(e_{i}^{\prime}\right)=e_{i}^{\prime}+\sum_{j} c_{i j} e_{j} .
$$

Let us choose $\lambda$ so that $C$ is the identity matrix and $a_{i}=e_{i}^{\prime}$. Then we have

$$
\tau([\lambda,[\lambda,[\lambda, h]]])=6 e_{1}^{\prime} \wedge e_{2}^{\prime} \wedge e_{3}^{\prime} \neq 0
$$

This completes the proof of Proposition 5.6. $\quad$ q.e.d.

Remark 5.8. It is not clear whether $\left(\mathcal{L}_{g, 1}\right)_{4} \subseteq \mathcal{K}_{g, 1}$.

\section{References}

[1] D. Bar-Natan, On the Vassiliev knot invariants, Topology 34 (1995) 423-472.

[2] D. Bar-Natan, S. Garoufalidis, L. Rozansky \& D. Thurston, The Arhus integral of rational homology 3-spheres, I-IV, Preprint, 1997. 
[3] S. Garoufalidis, On finite type 3-manifold invariants I, J. Knot Theory Ramifications 5 (1996) 441-462.

[4] S. Garoufalidis \& N. Habegger, The Alexander polynomial and finite type 3-manifolds invariants, Preprint, 1997.

[5] S. Garoufalidis \& J. Levine, On finite type 3-manifold invariants II, Math. Ann. 306 (1996) 691-718.

[6] - On finite type 3-manifold invariants IV: comparison of definitions, Math. Proc. Cambridge Philos. Soc. 122 (1997) 291-300.

[7] - Finite type 3-manifold invariants and the Torelli group. I, Invent. Math., in press.

[8] S. Garoufalidis \& T. Ohtsuki, On finite type 3-manifold invariants III: manifold weight systems, Topology, in press.

[9] - On finite type 3-manifold invariants V: rational homology 3-spheres, Proc. Aarhus Conf., Geometry and Physics, Marcel Dekker, New York, 1996, 445-457.

[10] R. Hain, Completions of the mapping class groups and the cycle $C-C^{-}$, Contemporary Math. 150 (1993) 75-105.

[11] _ Infinitesimal presentations of the Torelli groups, J. Amer. Math. Soc., in press.

[12] D. Johnson, Homeomorphisms of a surface which act trivially on homology, Proc. Amer. Math. Soc. 75 (1979) 118-125.

[13] _ A survey of the Torelli group, Contemporary Math. 20 (1983) 163-179.

[14] J. Hoste, A formula for Casson's invariant, Trans. Amer. Math. Soc. 297 (1986) 547-562.

[15] T. Kohno, Holonomy Lie algebras, logarithmic connections and the lower central series of fundamental groups, Contemporary Math. 90 (1990) 171-181.

[16] K. H. Ko, Seifert matrices and boundary link cobordisms, Trans. Amer. Math. Soc. 299 (1987) 657-681.

[17] T .T. Q. Le, J. Murakami \& T. Ohtsuki, A universal quantum invariant of 3manifolds, Topology, in press.

[18] T. T. Q. Le, An invariant of integral homology 3-spheres which is universal for all finite type invariants, Preprint, January 1996.

[19] J. Levine, Surgery equivalence of links, Topology 26 (1987) 45-61.

[20] S. Morita, Casson's invariant for homology 3-spheres and characteristic classes of vector bundles I, Topology 28 (1989) 305-323. 
[21] On the structure of the Torelli group and the Casson invariant, Topology 30 (1991) 603-621.

[22] T. Ohtsuki, Finite type invariants of integral homology 3-spheres, J. Knot Theory Ramification 5 (1996) 101-115.

[23] D. Quillen, On the associated graded ring of a group ring, J. Algebra 10 (1968) 411-418.

[24] D. Rolfsen, Knots and links, Publish or Perish, Houston, 1976.

[25] E. Witten, Quantum field theory and the Jones polynomial, Comm. Math. Phys. 121 (1989) 360-376.

[26] E. Witten \& J. Polchinski, Evidence for heterotic-type I string duality, Nuclear Phys. B460 (1996) 525.

HARVARD UNIVERSITY

BRANDEIS UNIVERSITY 